\title{
Three-Dimensional Numerical Modeling of Acoustic Trapping in Glass Capillaries
}

\author{
Ley, Mikkel Wennemoes Hvitfeld; Bruus, Henrik
}

Published in:

Physical Review Applied

Link to article, DOI:

10.1103/PhysRevApplied.8.024020

Publication date:

2017

Document Version

Publisher's PDF, also known as Version of record

Link back to DTU Orbit

Citation $(A P A)$ :

Ley, M. W. H., \& Bruus, H. (2017). Three-Dimensional Numerical Modeling of Acoustic Trapping in Glass

Capillaries. Physical Review Applied, 8(2), [024020 ]. https://doi.org/10.1103/PhysRevApplied.8.024020

\section{General rights}

Copyright and moral rights for the publications made accessible in the public portal are retained by the authors and/or other copyright owners and it is a condition of accessing publications that users recognise and abide by the legal requirements associated with these rights.

- Users may download and print one copy of any publication from the public portal for the purpose of private study or research.

- You may not further distribute the material or use it for any profit-making activity or commercial gain

- You may freely distribute the URL identifying the publication in the public portal

If you believe that this document breaches copyright please contact us providing details, and we will remove access to the work immediately and investigate your claim. 


\title{
Three-Dimensional Numerical Modeling of Acoustic Trapping in Glass Capillaries
}

\author{
Mikkel W. H. Ley ${ }^{*}$ and Henrik Bruus ${ }^{\dagger}$ \\ Department of Physics, Technical University of Denmark, \\ DTU Physics Building 309, DK-2800 Kongens Lyngby, Denmark \\ (Received 13 April 2017; published 22 August 2017)
}

\begin{abstract}
Acoustic traps are used to capture and handle suspended microparticles and cells in microfluidic applications. A particular simple and much-used acoustic trap consists of a commercially available, millimeter-sized, liquid-filled straight glass capillary actuated by a piezoelectric transducer. Here, we present a three-dimensional numerical model of the acoustic pressure field in the liquid coupled to the displacement field of the glass wall, taking into account mixed standing and traveling waves as well as absorption. The model explains the dynamical mechanism that leads to the formation of localized acoustic resonance modes in such a straight acoustic waveguide without any geometrical cavities in the axial direction of the capillary. The model further predicts that some of these modes are well suited for acoustic trapping, and it provides estimates for their frequencies and quality factors, the magnitude of the acoustic radiation force on a single test particle as a function of position, and the resulting acoustic retention force of the trap. We show that the model predictions are in agreement with published experimental results, and we discuss how improved and more-stable acoustic-trapping modes might be obtained using the model as a design tool.
\end{abstract}

DOI: 10.1103/PhysRevApplied.8.024020

\section{INTRODUCTION}

Microscale acoustofluidic devices are used increasingly in biology, the environmental and forensic sciences, and clinical diagnostics [1,2]. Examples include cell synchronization [3], the enrichment of prostate cancer cells in blood [4], the manipulation of C. elegans [5], and single-cell patterning [6]. Acoustics can also be used for noncontact microfluidic trapping and particle enrichment [7-9], as well as acoustic tweezing [10-14]. Trapping, an important unit operation in sophisticated celland bioparticle-handling systems, can also be obtained using other technologies, such as those in hydrodynamic [15], electro- and dielectrophoretic [16], and opticaltrapping systems [17]; see the review by Nilsson et al. [18]. However, one of the key parameters for successful commercialization of these technologies is throughput, and here only the hydrodynamic and acoustic systems are competitive [19]. Hydrodynamic systems offer simple device designs and can support high flow rates, as they rely solely on passive transport from inertial migration and drag induced by secondary flows [15]. However, they do not have the capabilities of active transport.

By contrast, acoustofluidics does support active migration, basically due to the acoustic contrast between the suspending medium and the particles [20], and it is therefore an inherently label-free technique. Moreover, it exhibits high cell viability, even over several days [21]. These

*mley@fysik.dtu.dk

†bruus@fysik.dtu.dk aspects and other appealing traits have increased the interest in acoustofluidic systems in recent years [1,2].

A subclass of acoustofluidic devices is based on simple, cheap, and commercially available glass capillaries. Early studies from the 1990s include improving agglutination between erythrocytes [22], simple noncontact particle manipulation in a circular wave tube by acoustic streaming and radiation forces [23], and highly sensitive size-selective particle separation [24]. However, the ultrasound capillary system remains an active research topic, as cell-handling applications have become more numerous and refined over the years. Recent examples include cardiac myoblast viability in ultrasonic fields [25], sonoporation [26], the squeezing of red blood cells [27], and the trapping and retention of particles against an external flow [28].

In this paper, we study specifically a millimeter-sized glass-capillary system used as a versatile acoustic trap in many experimental studies [8,9,26,27,29] and clinical applications, such as the isolation of cell-secreted membrane vesicles [30] and the capture and enrichment of bacteria from blood samples for rapid sepsis diagnostics [31]. A sketch of a generic capillary system is shown in Fig. 1, while typical material parameters are listed in Table I. The capillary is typically actuated locally by a piezoelectric transducer, which is coupled to the capillary either by epoxy glue or by a small drop of glycerol, the latter allowing for the removal and the reuse of both the capillary and the transducer. The device is driven at a resonance frequency to obtain the largest possible acoustic field in the fluid. In the optimal case, the main component of the resonance field is a vertically standing bulk acoustic 


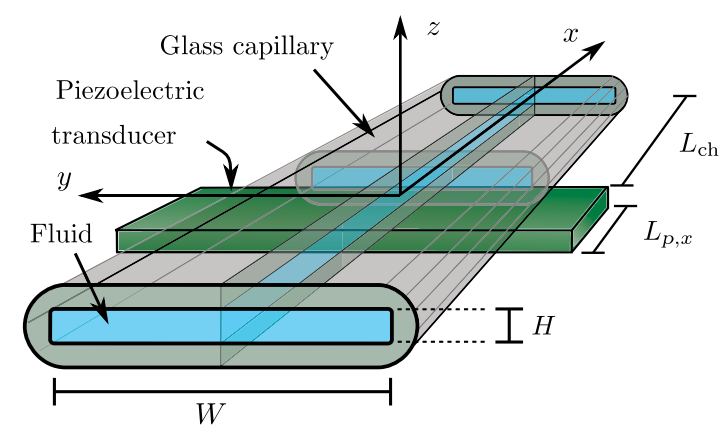

FIG. 1. A sketch of a generic capillary system for acoustic trapping. A millimeter-sized glass capillary (gray) filled with water (blue), is attached to a piezoelectric transducer (green) operated in the megahertz range. At certain frequencies, a resonant acoustic field builds up, in which case the acoustic radiation forces are strong enough to trap suspended microparticles in all spatial directions, and even to retain them against drag from an axial fluid flow through the capillary.

wave (SBAW) along the $z$ direction, which unavoidably is mixed with a traveling bulk acoustic wave (TBAW) in the axial $x$ direction along the capillary away from the transducer region.

The resulting acoustic radiation forces trap particles above or near the transducer in all spatial directions and are often strong enough to retain a single microparticle against the drag from a fluid flow.

In contemporary acoustofluidics, it is a challenge to model and optimize the design of a given device. Examples of recent advances in modeling include Lei et al. [29], who

TABLE I. Material parameters at $25^{\circ} \mathrm{C}$ and $4 \mathrm{MHz}$ used in the numerical modeling of the capillary acoustic trap.

\begin{tabular}{lccc}
\hline \hline Parameter & Symbol & Value & Unit \\
\hline Water [32]: & & & \\
Mass density & $\rho_{\mathrm{fl}}$ & 997.05 & $\mathrm{~kg} \mathrm{~m}^{-3}$ \\
Compressibility & $\kappa_{\mathrm{fl}}$ & 452 & $\mathrm{TPa}^{-1}$ \\
Speed of sound & $c_{\mathrm{fl}}$ & 1496.7 & $\mathrm{~m} \mathrm{~s}^{-1}$ \\
Dynamic viscosity & $\eta_{\mathrm{fl}}$ & 0.890 & $\mathrm{mPa} \mathrm{s}$ \\
Damping coefficient [33] & $\Gamma_{\mathrm{fl}}$ & 0.004 & \\
Viscous boundary layer & $\delta$ & 0.27 & $\mu \mathrm{m}$ \\
Pyrex glass [34]: & & & \\
Mass density & $\rho_{\mathrm{sl}}$ & 2230 & $\mathrm{~kg} \mathrm{~m}^{-3}$ \\
Young's modulus & $E$ & 62.75 & $\mathrm{GPa}^{-3}$ \\
Poisson's ratio & $\nu$ & 0.2 & \\
Speed of sound, longitudinal & $c_{\mathrm{lo}}$ & 5592 & $\mathrm{~m} \mathrm{~s}^{-1}$ \\
Speed of sound, transverse & $c_{\mathrm{tr}}$ & 3424 & $\mathrm{~m} \mathrm{~s}^{-1}$ \\
Damping coefficient [33] & $\Gamma_{\mathrm{sl}}$ & 0.0004 & \\
Polystyrene (ps) for 12- $\mu \mathrm{m}-$ diameter ps particles in & water: \\
Mass density [35] & $\rho_{\mathrm{ps}}$ & 1050 & $\mathrm{~kg} \mathrm{~m}^{-3}$ \\
Compressibility [36] & $\kappa_{\mathrm{ps}}$ & 249 & $\mathrm{TPa}^{-1}$ \\
Poisson's ratio [37] & $\nu_{\mathrm{ps}}$ & 0.35 & \\
Speed of sound at 20 ${ }^{\circ}$ C [38] & $c_{\mathrm{ps}}$ & 2350 & $\mathrm{~m} \mathrm{~s}^{-1}$ \\
Monopole coefficient, Eq. (6b) & $f_{0}$ & 0.48 & \\
Dipole coefficient, Eq. (6c) & $f_{1}$ 0.052-0.003i & \\
\hline \hline
\end{tabular}

modeled the three-dimensional (3D) fluid domain without taking the solid domain into account; Muller and Bruus [32,39], who made detailed models in 2D of the thermoviscous and transient effects in the fluid domain; Gralinski et al. [28], who modeled circular capillaries in 3D with fluid and glass domains without taking absorption and outgoing waves into account; Hahn and Dual [33], who calculated the acoustic field in a 3D model for a glass-silicon device (not a capillary system) and characterized the various loss mechanisms; and Garofalo et al. [40], who studied a coupled transducer-silicon-glass-water system in 2D.

In this work, we present a 3D numerical model of the capillary acoustic trap of Fig. 1. We model the acoustic pressure field in the liquid coupled to the displacement field of the glass wall, taking into account mixed standing and traveling waves as well as absorption. We model the outlets, which, in practice, are connected to long tubes, either as being free reflecting surfaces or perfect absorbers of outgoing acoustic waves. Our model explains the dynamical mechanism that leads to the formation of localized acoustic resonance modes in the straight capillaries that are without geometrical cavities in the axial $x$ direction. Furthermore, we compare prior experimental results from the four devices listed in Table II with predictions of our model, particularly the frequency response, the levitating resonance modes, and the acoustic-trapping forces. For one of the devices, we perform two convergence analyses to show to which degree numerical convergence is obtained. Lastly, we demonstrate how to apply the model as a design tool by studying the effects of narrowing the width of a given capillary.

\section{THEORY AND NUMERICAL MODEL}

We model single-frequency harmonic actuation at frequency $f$ and angular frequency $\omega=2 \pi f$, such that any first-order acoustic field $g$ in the complex-phase representation has the time dependence $g(\boldsymbol{r}, t)=g(\boldsymbol{r}) e^{-i \omega t}$.

Following the thorough analysis by Hahn and Dual [33], we introduce effective absorption in our equations by modifying the time derivative as $\partial_{t} \rightarrow-i \omega(1+i \Gamma)$, where $\Gamma \ll 1$ is an effective absorption parameter, with values for the respective materials listed in Table I. The remaining details of the model are explained in the following subsections.

\section{A. Governing equations}

The dynamics of the solid (Pyrex) is modeled by the elastic displacement $\boldsymbol{u}$, the stress $\boldsymbol{\sigma}_{\mathrm{sl}}$, the density $\rho_{\mathrm{sl}}$, and the transverse and longitudinal speeds of sound, $c_{\mathrm{tr}}$ and $c_{\mathrm{lo}}$,

$$
\begin{aligned}
& \boldsymbol{\nabla} \cdot \boldsymbol{\sigma}_{\mathrm{sl}}=-\rho_{\mathrm{sl}} \omega^{2}\left(1+i \Gamma_{\mathrm{sl}}\right)^{2} \boldsymbol{u}, \\
& \frac{1}{\rho_{\mathrm{sl}}} \boldsymbol{\sigma}_{\mathrm{sl}}=c_{\mathrm{tr}}^{2}\left[\boldsymbol{\nabla} \boldsymbol{u}+(\boldsymbol{\nabla} \boldsymbol{u})^{T}\right]+\left(c_{\mathrm{lo}}^{2}-2 c_{\mathrm{tr}}^{2}\right)(\boldsymbol{\nabla} \cdot \boldsymbol{u}) \mathbf{I},
\end{aligned}
$$


TABLE II. Capillary geometries modeled in this work: $C 1$ from Hammarström et al. [8], $C 2$ from Lei et al. [29], $C 3$ from Mishra et al. [27], $C 4$ from Gralinski et al. [28], and $C 5$ a capillary design proposed in this work. Besides the symbols defined in Fig. $2, R_{\mathrm{cu}}$ is the radius of curvature of the fluid-channel corners, $f_{\text {res }}^{\exp }$ and $f_{\text {res }}$ is the experimental and numerical resonance frequencies, respectively, and $Q$ is the numerically calculated quality factor.

\begin{tabular}{lccccccccccc}
\hline \hline Device & $L[\mathrm{~mm}]$ & $W[\mathrm{~mm}]$ & $H[\mathrm{~mm}]$ & $H_{\mathrm{gl}}[\mathrm{mm}]$ & $R_{\mathrm{cu}}[\mu \mathrm{m}]$ & $L_{p, x}[\mathrm{~mm}]$ & $L_{p, x}[\mathrm{~mm}]$ & $f_{\text {res }}^{\mathrm{exp}}[\mathrm{MHz}]$ & $f_{\text {res }}[\mathrm{MHz}]$ & $Q$ & $d_{0}[\mathrm{~nm}]$ \\
\hline$C 1$ & 2.0 & 2.0 & 0.20 & 0.14 & 25 & 1.16 & 2.0 & 3.970 & 3.906 & 53 & 0.10 \\
$C 2$ & 2.0 & 6.0 & 0.30 & 0.30 & 25 & 1.0 & 1.0 & 2.585 & 2.495 & 78 & 0.10 \\
$C 3$ & 8.5 & 0.1 & 0.10 & 0.05 & 19 & 15 & 1.0 & 7.900 & 6.406 & 222 & 0.10 \\
$C 4$ & 10.0 & 0.85 & 0.85 & 0.225 & 425 & 4.0 & 1.0 & 1.055 & 0.981 & 109 & 0.10 \\
$C 5$ & 2.0 & 0.5 & 0.20 & 0.14 & 25 & 1.16 & 0.50 & $\cdots$ & 4.201 & 53 & 0.10 \\
\hline \hline
\end{tabular}

$$
c_{\mathrm{tr}}^{2}=\frac{1}{2(1+\nu)} \frac{E}{\rho_{\mathrm{sl}}}, \quad c_{\mathrm{lo}}^{2}=2 c_{\mathrm{tr}}^{2} \frac{1-\nu}{1-2 \nu}
$$

Here, I is the unit tensor, $E$ is Young's modulus, and $\nu$ is Poisson's ratio. The fluid (water) with its acoustic pressure $p$, velocity $\boldsymbol{v}$, density $\rho_{\mathrm{fl}}$, and sound speed $c_{\mathrm{fl}}$ is modeled as pressure acoustics with absorption [33],

$$
\begin{aligned}
\nabla^{2} p & =-\frac{\omega^{2}}{c_{\mathrm{fl}}^{2}}\left(1+i \Gamma_{\mathrm{fl}}\right)^{2} p, \\
\boldsymbol{v} & =\frac{-i}{\omega \rho_{\mathrm{fl}}} \nabla p .
\end{aligned}
$$

\section{B. Boundary conditions}

The applied boundary conditions are summarized in Table III and Fig. 2. Because the $y-z$ and $x-z$ planes are mirror planes, we model only a quarter of the system, and symmetry conditions are applied on the two symmetry planes. The stress is zero on all outer boundaries facing the air, and the stress and the velocity fields are continuous across all internal boundaries.

The model of the piezoelectric actuation on the interface $\partial \Omega_{\mathrm{pz}}$ is not straightforward, as neither the excited modes of the piezoelectric transducer nor the coupling layer by which the transducer is attached to the glass capillary have been characterized experimentally in the published studies. We therefore choose to explore the effects of two idealized generic actuation conditions that mimic a simple uniform contraction-expansion mode that pushes and pulls on the

TABLE III. Boundary conditions imposed on the solid and fluid domains in the model shown in Fig. 2.

\begin{tabular}{ll}
\hline \hline Domain $\leftarrow$ boundary & Boundary condition \\
\hline Solid domain $\leftarrow$ air & $\boldsymbol{\sigma}_{\mathrm{sl}} \cdot \boldsymbol{n}=\mathbf{0}$ \\
Solid domain $\leftarrow$ fluid & $\boldsymbol{\sigma}_{\mathrm{sl}} \cdot \boldsymbol{n}=-p \boldsymbol{n}$ \\
Solid domain $\leftarrow$ transducer & $\mathrm{Eq} .(3 \mathrm{a})$ or Eq. $(3 \mathrm{~b})$ \\
Solid domain $\leftarrow$ symmetry & $\boldsymbol{u} \cdot \boldsymbol{n}=0, \boldsymbol{t} \cdot \boldsymbol{\sigma}_{\mathrm{s} 1} \cdot \boldsymbol{n}=0$ \\
Fluid domain $\leftarrow$ solid & $\boldsymbol{v} \cdot \boldsymbol{n}=-i \omega \boldsymbol{u} \cdot \boldsymbol{n}$ \\
Fluid domain $\leftarrow$ air & $p=0$ \\
Fluid domain $\leftarrow$ symmetry & $\boldsymbol{n} \cdot \boldsymbol{\nabla} p=0$ \\
\hline \hline
\end{tabular}

glass-capillary surface through a coupling layer consisting of either a rigid epoxy glue or a fluid glycerol film. From previous work [36], we know that a wall displacement amplitude $d_{0}=0.1 \mathrm{~nm}$ leads to realistic values for experimentally measured pressures [41-43], so we use this value throughout this work. To mimic the rigid epoxy glue, we assume a simple uniform displacement amplitude $u_{z}=d_{0}$ in the $z$ direction without transverse motion, Eq. (3a), while, to mimic the fluid glycerol layer with a typical thickness of 10-100 $\mu \mathrm{m}$ that can easily accommodate nanometer-sized fluctuations as well as transverse displacement, we assume that only the area average $\left\langle u_{z}\right\rangle_{\mathrm{pz}}$ of the normal displacement $u_{z}$ is $d_{0}$, while the shear stress is zero, Eq. (3b),

$$
u_{z}=d_{0}, \quad u_{x}=u_{y}=0, \quad \text { rigid displacement }
$$

$\left\langle u_{z}\right\rangle_{\mathrm{pz}}=d_{0}, \sigma_{x z}=\sigma_{y z}=0$, average displacement.

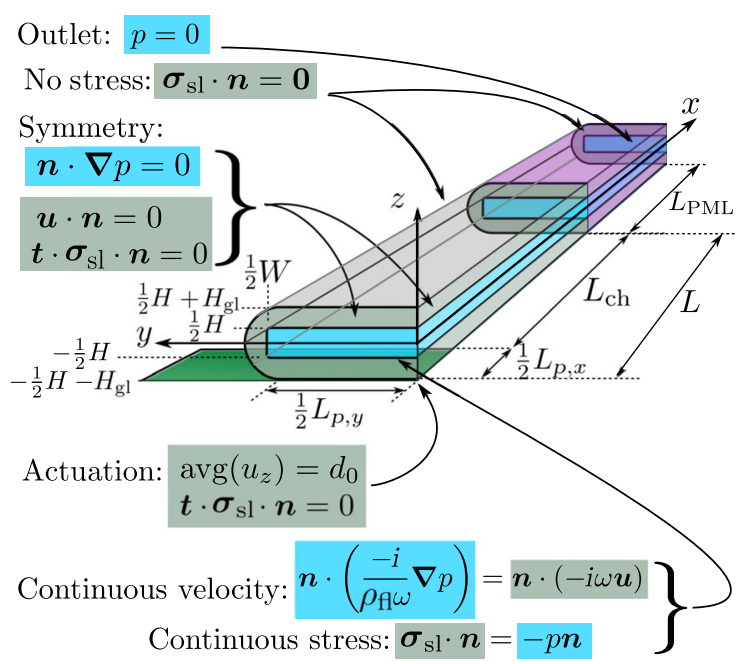

FIG. 2. The computational domain used in the numerical model of Fig. 1 reduced to the quadrant $x>0$ and $y>0$ due to symmetry. Shown are the solid wall (gray), the water channel (blue), the actuation plane (green), the perfectly matched layer (PML, purple), and arrows allocating the boundary conditions to their respective boundaries. 


\section{The outlet and perfectly matched layers}

In most setups, the capillary is connected to the microfluidic circuit through tubings at the ends. If a large section of the capillary is inserted into a connecting elastic tube [29], this might cause significant absorption. Furthermore, the water domain continues uninterrupted from the capillary into the connecting tube, so an extreme case is that none of the acoustic waves going out from the actuation region are reflected at the ends of the capillary. We model this situation using a perfectly matched layer (PML), which is a domain acting as an artificial perfect absorber of all outgoing TBAWs along the $x$ axis. In our model, the region $x<L$ is normal, while the PML of length $L_{\mathrm{PML}}$ is at $L<x<$ $L+L_{\mathrm{PML}}$; see Fig. 2. Following Collino and Monk [44], ideal absorption along the $x$ direction for $x>L$ can be obtained by a complex-valued coordinate stretching of $x$ in the PML domain. This stretching is based on a real-valued function $s(x)$, which is zero in the normal region $x<L$ and increases smoothly from zero in the PML as

$s(x)=k_{\mathrm{PML}}\left(\frac{x-L}{L_{\mathrm{PML}}}\right)^{2}, \quad$ for $L \leq x \leq L+L_{\mathrm{PML}}$,

where $k_{\mathrm{PML}}>0$ is the absorption strength. The coordinate stretching is implemented in the model by changing all occurrences of $\partial_{x}$ and the integral measure $d x$ as

$$
\begin{aligned}
\partial_{x} \rightarrow \partial_{\tilde{x}} & =\frac{1}{1+i s(x)} \partial_{x}, \\
d x \rightarrow d \tilde{x} & =[1+i s(x)] d x,
\end{aligned}
$$

where the latter appears in the weak-form implementation of the governing equations and boundary conditions; see Sec. II E. The values of $L_{\mathrm{PML}}$ and $k_{\mathrm{PML}}$ are also discussed in Sec. II E. The coordinate stretching in the PML introduces a positive imaginary part in the wave numbers in the $x$ direction, and it thus dampens the outgoing waves exponentially along the $x$ axis.

The opposite limit of zero absorption is handled by dropping the PML and imposing the no-stress condition at $x=L$.

\section{Acoustic energy density and radiation force}

Good acoustic trapping in a capillary requires a high acoustic energy density $E_{\mathrm{ac}}^{\mathrm{fl}}$ in the fluid [45] and $E_{\mathrm{ac}}^{\mathrm{sl}}$ in the solid [46], both given by the sum of the space- and timeaveraged kinetic and potential energy density, in the respective volumes $V_{\text {act }}^{\mathrm{fl}}$ and $V_{\text {act }}^{\mathrm{sl}}$ above the transducer,

$$
\begin{aligned}
& E_{\mathrm{ac}}^{\mathrm{fl}}=\int_{V_{\mathrm{act}}^{\mathrm{fl}}}\left(\frac{1}{2} \rho_{\mathrm{fl}}\left\langle v^{2}\right\rangle+\frac{1}{2} \kappa_{\mathrm{fl}}\left\langle p^{2}\right\rangle\right) \frac{d V}{V_{\mathrm{act}}^{\mathrm{fl}}}, \\
& E_{\mathrm{ac}}^{\mathrm{sl}}=\int_{V_{\mathrm{act}}^{\mathrm{sl}}}\left\{\frac{1}{2} \rho_{\mathrm{sl}} \omega^{2}\left\langle u^{2}\right\rangle+\frac{1}{4}\left\langle\boldsymbol{\sigma}_{\mathrm{sl}}:\left[\nabla \boldsymbol{u}+(\nabla \boldsymbol{u})^{T}\right]\right\rangle\right\} \frac{d V}{V_{\mathrm{act}}^{\mathrm{sl}}},
\end{aligned}
$$

where each set of angled brackets denotes the time average over one acoustic oscillation period.

The trapping force acting on an elastic particle of radius $a_{\mathrm{pa}}$, density $\rho_{\mathrm{pa}}$, and compressibility $\kappa_{\mathrm{pa}}$ suspended in the fluid is the acoustic radiation force $\boldsymbol{F}^{\mathrm{rad}}$, which, for mixed standing and traveling waves, is given by [47]

$\boldsymbol{F}^{\mathrm{rad}}=-\frac{4}{3} \pi a_{\mathrm{pa}}^{3}\left[\kappa_{\mathrm{fl}}\left\langle\left(f_{0} p\right) \boldsymbol{\nabla} p\right\rangle-\frac{3}{2} \rho_{\mathrm{fl}}\left\langle\left(f_{1} \boldsymbol{v}\right) \cdot \boldsymbol{\nabla} \boldsymbol{v}\right\rangle\right]$,

where the monopole and dipole scattering coefficients $f_{0}$ and $f_{1}$, respectively, are

$$
\begin{aligned}
& f_{0}=1-\frac{\kappa_{\mathrm{pa}}}{\kappa_{\mathrm{fl}}}, \\
& f_{1}=\frac{2(1-\gamma)\left(\frac{\rho_{\mathrm{pa}}}{\rho_{\mathrm{fl}}}-1\right)}{2 \frac{\rho_{\mathrm{\rho a}}}{\rho_{\mathrm{fl}}}+1-3 \gamma}, \quad \gamma=-\frac{3}{2}[1+i(1+\tilde{\delta})] \tilde{\delta},
\end{aligned}
$$

with $\tilde{\delta}=\left[1 /\left(a_{\mathrm{pa}}\right)\right] \sqrt{\left[\left(2 \eta_{\mathrm{fl}}\right) / \omega \rho_{\mathrm{fl}}\right]}$ being the viscous boundary-layer thickness normalized by the particle radius $a_{\mathrm{pa}}$.

\section{E. Numerical implementation in weak form}

Following Gregersen et al. [48], we implement the governing equations and boundary conditions in the weakform partial-differential-equation module of the finiteelement software COMSOL MULTIPHYSICS 5.2 [49]. We use Lagrangian test functions of second order for $p, u_{x}$, $u_{y}$, and $u_{z}$, and we solve for given geometry, materials, actuation frequency $\omega$, and actuation displacement $d_{0}$.

We test the PML for capillary $C 1$ as follows; see Fig. 3. The length $L_{\mathrm{PML}}$ of the PML is comparable to the longest wavelength of the system [50], here the longitudinal wavelength $\lambda_{\mathrm{lo}}=2 \pi c_{\mathrm{lo}} / \omega=1.4 \mathrm{~mm}$ in Pyrex at $4 \mathrm{MHz}$. By running parametric sweeps in $L_{\mathrm{PML}} \approx \lambda_{\mathrm{lo}}$ and $k_{\mathrm{PML}}>0$, we find that, for an $L_{\mathrm{PML}}$ as short as $0.25 \lambda_{\mathrm{lo}}$, the results are independent of the PML strength in the range $10<k_{\mathrm{PML}}<10^{4}$, showing that for this broad parameter range, the outgoing waves are absorbed before reaching the PML back edge at $x=L+L_{\mathrm{PML}}$, and without traces of backscattering from the PML front edge at $x=L$. To further show the independence of the results on the PML, we set $L_{\mathrm{PML}}=0.25 \lambda_{\mathrm{lo}}$ and $k_{\mathrm{PML}}=10^{3}$, and we vary the length $L_{\mathrm{ch}}$ of the capillary between the piezoelectric transducer and the PML front edge. In Fig. 3, $p$ and $u_{z}$ are plotted versus $x$ for four fixed $(y, z)$ coordinates, and it is seen that, for $L_{\mathrm{ch}} \gtrsim \lambda_{\mathrm{lo}}$, the resulting fields coincide everywhere outside the PML domain. For smaller values $L_{\mathrm{ch}} \lesssim \lambda_{\mathrm{lo}}$, we do observe deviations in the response, indicating that the outgoing waves are not fully established before they enter the PML and are absorbed. Consequently, in the following, we choose the fixed parameter values $L_{\mathrm{ch}}=1 \mathrm{~mm}, L_{\mathrm{PML}}=0.25 \lambda_{\mathrm{lo}}=$ $0.35 \mathrm{~mm}$, and $k_{\mathrm{PML}}=10^{3}$. 

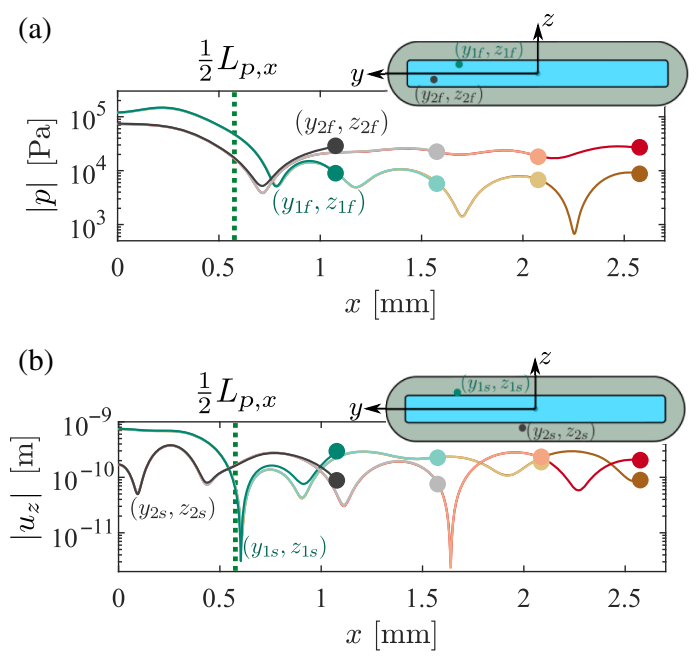

FIG. 3. The acoustic field in capillary $C 1$ as a function of the front-edge position $L=\frac{1}{2} L_{p, x}+L_{\mathrm{ch}}$ of the PML domain with fixed $L_{\mathrm{PML}}=0.25 \lambda_{L}$ and $k_{\mathrm{PML}}=10^{3}$. (a) The pressure amplitude $|p|$ versus $x$ (the colored lines) terminating at $L$ (the colored points) for the two $(y, z)$ positions $\left(y_{1 f}, z_{1 f}\right)=(0.3 W, 0.35 H)$ and $\left(y_{2 f}, z_{2 f}\right)=(0.4 W,-0.25 H)$ (the green and black points in the inset). The line colors correspond to $L_{\mathrm{ch}}=0.5,1.0,1.5$, and $2.0 \mathrm{~mm}$ from green (black) to brown (red). The vertical green dotted line at $x=\frac{1}{2} L_{p, x}$ marks the edge of the actuation region. (b) Similar plot for the vertical displacement amplitude $\left|u_{z}\right|$, but here for the two $(y, z)$ positions $\left(y_{1 s}, z_{1 s}\right)=(0.3 W, 0.6 H)$, and $\left(y_{2 s}, z_{2 s}\right)=(0.05 W,-0.7 H)$.

The mesh is generated by first defining a mesh in the $y-z$ plane [see Fig. 4(a)] and then extruding it equidistantly along the $x$ axis using the "swept-mesh" function in COMSOL MULTIPHYSICS; see Fig. 4(b). The size of the mesh elements is controlled by the maximum mesh size $d_{\text {mesh }}$, except for elements on the $z$ axis (the intersection of the two symmetry planes) and on the corner edges of the fluid channel (where the curvature is large), both of which are assigned a smaller maximum mesh size. All mesh sizes used in the model are listed in Table IV. Unless stated otherwise, we have set $d_{\text {mesh }}=H / 7$.

For a given field variable $g$, we perform a meshconvergence test based on the convergence parameter $C(g)$, which is defined in Ref. [36] as

$$
C(g)=\sqrt{\frac{\int_{\Omega} d V\left|g-g_{\mathrm{ref}}\right|^{2}}{\int_{\Omega} d V\left|g_{\mathrm{ref}}\right|^{2}}}, \quad \text { for } 0 \leq x \leq L .
$$

Here, the integration volume $\Omega$ is the domain in which $g$ is defined but excludes the PML domain. The field $g$ is calculated with the abovementioned characteristic mesh size $d_{\text {mesh }}$, and $g_{\text {ref }}$ is the reference field calculated with the finer mesh $d_{\text {mesh }}^{\text {ref }}=0.9 d_{\text {mesh }}$. We cannot use a smaller value of $d_{\text {mesh }}^{\text {ref }}$ because this value, combined with the length $L=2.5 \mathrm{~mm}$, results in a memory consumption of 85 gigabytes of RAM out of the 128 gigabytes available on

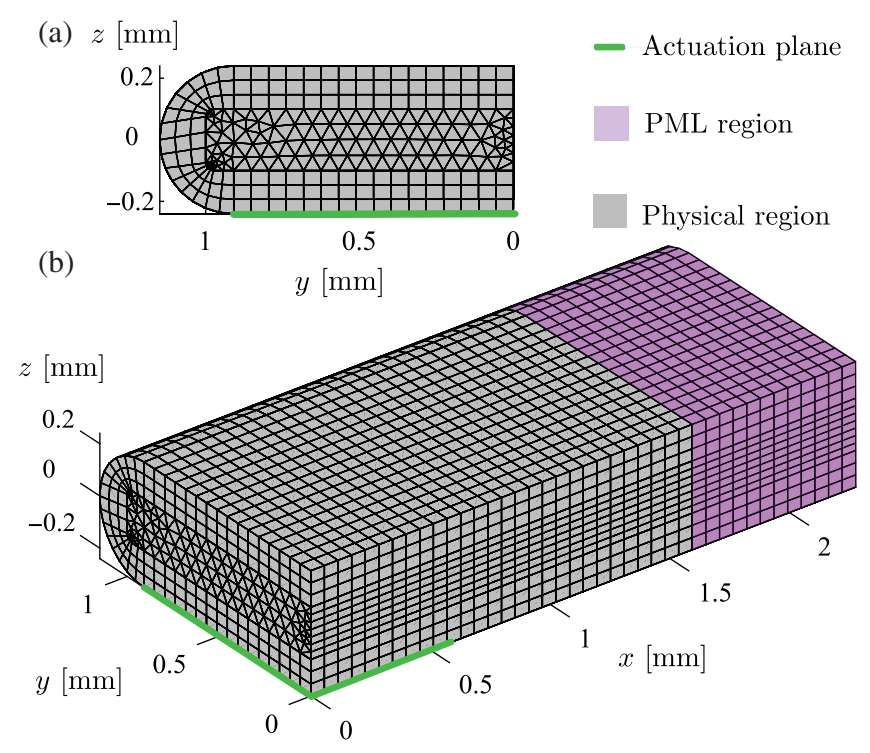

FIG. 4. A coarse version of the mesh at the surface of the computational domain of Fig. 2 for a mesh size of $d_{\text {mesh }}=$ $57.2 \mu \mathrm{m}$, which is twice the value used in most calculations; see Table IV. (a) The 2D mesh in the $y-z$ plane consisting of a structured mesh in the solid, a free triangular mesh in the fluid, and a refined mesh at the corners of the fluid domain as well as at the vertical symmetry line $y=0$. (b) The full 3D mesh generated by extruding the 2D mesh shown in (a) along the $x$ direction with equidistant spacing.

our workstation; see Sec. III. With these values, our simulation involves $2 \times 10^{6}$ degrees of freedom and a computation time of around 15 min per parameter set. In Fig. 5, semilog plots of $C$ versus $d_{\text {mesh }}^{\text {ref }} / d_{\text {mesh }}$ for all four fields are shown. The plots exhibit an exponential decrease of $C$, which indicates good numerical mesh convergence. For the chosen mesh sizes, we obtain $C \approx 0.002$, which is an acceptable level for the present study.

Finally, the area-averaged actuation condition (3b) is imposed on the displacement field $u_{z}$ on the actuation interface using an envelope function $F(x)$,

TABLE IV. List of the mesh parameters used in the model. The maximum mesh element size is set to $d_{\text {mesh }}=H / 7$, with three exceptions: (1) for the reference field $g_{\text {ref }}$ of $C 1$ in Eq. (7) and Fig. 5, where $d_{\text {mesh }}^{\text {ref }}=0.9 d_{\text {mesh }}$, (2) for Fig. 6, where $d_{\text {mesh }}=40.0 \mu \mathrm{m}$, and (3) for $C 4$, where $d_{\text {mesh }}=H / 20$.

\begin{tabular}{lccc}
\hline Location & Linear size & No. of elements & Mesh size \\
\hline$y-z$ plane: & & 7 & \\
Bulk fluid & $H$ & 6 & $d_{\text {mesh }}$ \\
Bulk solid & $H_{\mathrm{gl}}$ & 10 & $0.168 d_{\text {mesh }}$ \\
Corner solid fluid & $\frac{1}{2} \pi R_{\mathrm{cu}}$ & 15 & $0.5 d_{\text {mesh }}$ \\
Fluid symmetry edge & $H$ & & \\
$x$ direction (extruded): & & 80 & $d_{\text {mesh }}$ \\
Bulk fluid & $L+L_{\mathrm{PML}}$ & 80 & $d_{\text {mesh }}$ \\
Bulk solid & $L+L_{\mathrm{PML}}$ & &
\end{tabular}




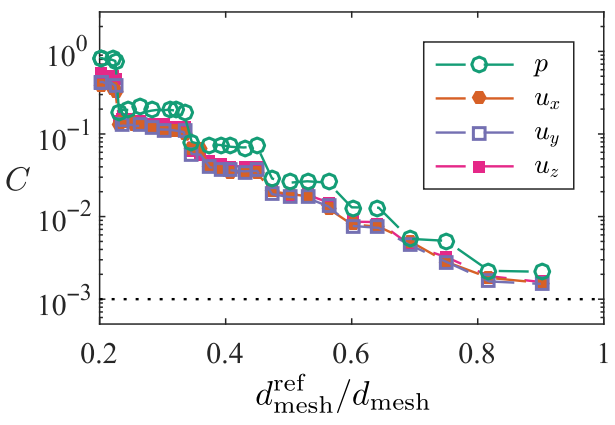

FIG. 5. Semilog plot of the convergence parameter $C$ defined in Eq. (7) versus $d_{\text {mesh }}^{\text {ref }} / d_{\text {mesh }}$, the inverse of the maximum mesh size. The mesh parameter values are listed in Table IV.

$$
\begin{gathered}
\int_{0}^{L_{p}, x / 2} \frac{2 d x}{L_{p, x}} \int_{0}^{L_{p}, y / 2} \frac{2 d y}{L_{p, y}}\left(u_{z}-d_{0}\right) F(x)=0, \\
\text { with } \quad F(x)=-\tanh \left(\frac{x-\frac{1}{2} L_{p}}{\Delta L_{p}}\right),
\end{gathered}
$$

where the transition length is set to $\Delta L_{p}=100 \mu \mathrm{m}$.

\section{RESULTS}

We characterize numerically the four different glass capillaries $C 1, C 2, C 3$, and $C 4$ listed in Table II, for which we find experimental results in the literature: $C 1$ from Hammarström et al. [8] (also employed in Refs. [7,9]), C2 from Lei et al. [29], $C 3$ from Mishra et al. [27], and $C 4$ from Gralinski et al. [28]. We also characterize a lowaspect-ratio version of $C 1$ denoted $C 5$. We simulate the acoustic fields for these five capillary systems, each with the two different actuation boundary conditions (3b) and (3a), and each both with and without the absorbing PML region. Supplementally to these studies, we simulate geometrical variations to study the effects of the capillaries' manufacturing tolerances and the length-dependent reflections from the capillary end. Consequently, we study more than 20 different capillary configurations, each swept in frequency using 50 values or more, resulting in over 1000 simulations, all of which show good numerical convergence without spurious effects. We remark that a simulation of a given frequency and geometry takes between 2 and 15 min on our workstation, a Dell Inc Precision T3610 Intel Xeon CPU E5-1650 v2 at $3.50 \mathrm{GHz}$ with 128 gigabytes of RAM and 6 CPU cores. To gain an overall understanding of these systems, we focus in the following subsections on different acoustofluidic aspects for each capillary.

\section{A. Analysis of capillary $\boldsymbol{C 1}$}

For capillary $C 1$ [8], we mimic the experimental use of glycerol to attach the transducer by applying the areaaveraged condition ( $3 b$ ) for the actuation. Moreover, to avoid the complications arising from reflections at the end

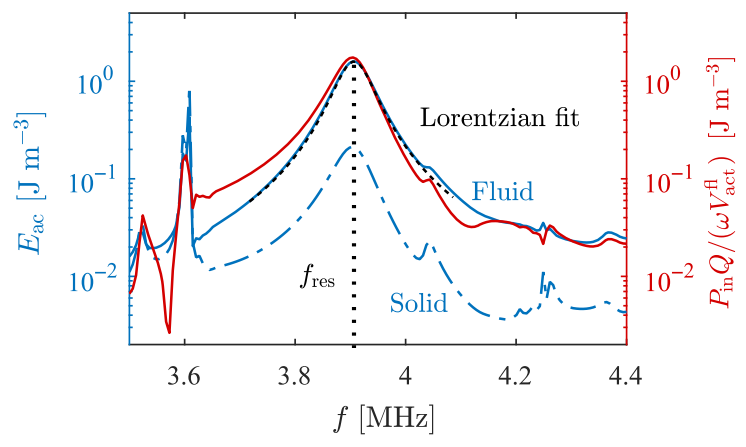

FIG. 6. Semilog plot of simulation results for capillary $C 1$ of the acoustic energy density $E_{\mathrm{ac}}$ (the left axis) in the fluid (the blue solid line) and in the solid (the blue dashed line) above the transducer, and the normalized power input $Q P_{\text {in }} /\left(\omega V_{\text {act }}^{\mathrm{fl}}\right)$ (the red line, right axis) from the transducer as a function of the frequency $f . E_{\text {ac }}$ is fitted well by a Lorentzian peak (the black dashed line) having a resonance frequency $f_{\text {res }}=3.906 \mathrm{MHz}$, a full-width-half-maximum linewidth of $\Delta f=0.7369 \mathrm{MHz}$, a quality factor of $Q=53.0$, and a maximum of $E_{\mathrm{ac}}=1.60 \mathrm{~J} / \mathrm{m}^{3}$.

of the capillary, we first assume perfect absorption of outgoing waves and thus use the PML.

First, we study the frequency response. In Fig. 6, we show a semilog plot of the volume-averaged acoustic energy density in the fluid volume above the transducer $\left(0<x<\frac{1}{2} L_{p, x}\right), E_{\mathrm{ac}}^{\mathrm{fl}}$ for the fluid volume $V_{\mathrm{act}}^{\mathrm{fl}}$, and $E_{\mathrm{ac}}^{\mathrm{sl}}$ for the solid volume $V_{\mathrm{act}}^{\mathrm{sl}}$, as a function of the actuation frequency $f$. A levitating resonance, strongly dominated by the energy density in the fluid, is identified at $f_{\text {res }}=3.906 \mathrm{MHz}$, only $2 \%$ lower then the experimental value $f_{\text {res }}^{\exp }=3.970 \mathrm{MHz}$ and $4 \%$ from the simple halfwave value $c_{\mathrm{fl}} /(2 H)=3.74 \mathrm{MHz}$. The resonance peak is fitted well by a Lorentzian line shape centered around $f_{\text {res }}=3.906 \mathrm{MHz}$ with a quality factor $Q=53.0$.

We successfully verify that this $Q$ factor is consistent with the relation $E_{\mathrm{ac}}^{\mathrm{tot}}=Q P_{\text {in }} /\left(\omega V_{\mathrm{act}}^{\mathrm{fl}}\right)$, where $P_{\text {in }}=$ $\int_{A_{\text {act }}}\left\langle(-i \omega \boldsymbol{u}) \cdot \boldsymbol{\sigma}_{\mathrm{sl}} \cdot \boldsymbol{n}\right\rangle d a$ is the time-averaged power delivered by the transducer, and $E_{\mathrm{ac}}^{\mathrm{tot}}=E_{\mathrm{ac}}^{\mathrm{fl}}+E_{\mathrm{ac}}^{\mathrm{sl}}$ is the total stored acoustic energy density. Here, we exploit that in a steady state, the input power equals the dissipated power. Besides the main levitating resonance peak, minor resonances without specific structure are also present, reflecting the many complex modes in the coupled fluid-solid system. None of these resonances have particularly good trapping properties, and they would probably not be observed in acoustophoretic experiments.

Next, we study the acoustic field. Upon inspection of the pressure and displacement fields at the resonance $f_{\text {res }}$, we find them to be mainly a standing wave above the actuation plane combined with an outgoing traveling wave away from the transducer region for $x>\frac{1}{2} L_{p, x}$; see Videos 1 and 2. An animated Graphics Interchange Format (GIF) version of each video in the paper can be found in the Supplemental Material [51]. The amplitude of the acoustic 


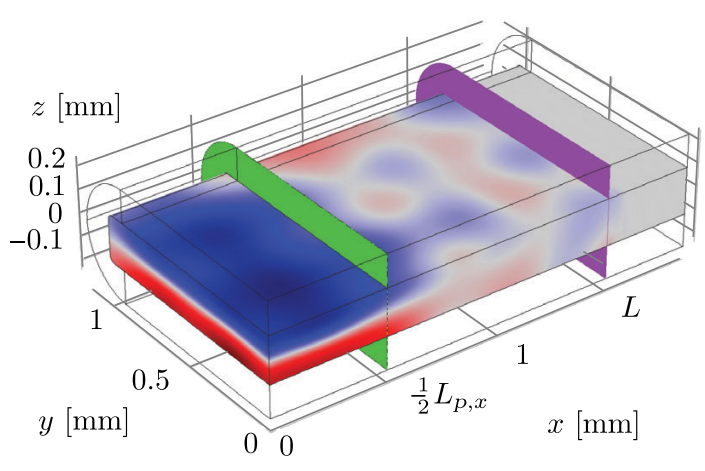

VIDEO. 1. Color plot of the pressure field $p$ in the fluid from $-0.18 \mathrm{MPa}$ (blue) to $0.18 \mathrm{MPa}$ (red) of capillary $C 1$ at the levitating half-wave resonance $f_{\text {res }}=3.906 \mathrm{MHz}$. The green and purple planes represent the end of the actuation region and the beginning of the PML domain, respectively.

fields $p$ and $\boldsymbol{u}$ are largest above the transducer. The fluid pressure $p$ is a nearly perfect vertical half wave with a horizontal nodal plane near the channel center, which enables microparticle levitation given its maximum of $0.19 \mathrm{MPa}$ for an actuation amplitude $d_{0}=0.1 \mathrm{~nm}$. Because of this feature, we refer to this type of resonance as levitating half-wave resonance in the following. At the edge of the transducer, the pressure amplitude drops an order of magnitude, which, according to Eq. (6a), gives rise to the lateral forces that constitute the acoustic trap.

For the displacement field in the solid, we find its maximum value to be $1.2 \mathrm{~nm}$, which is an order of magnitude larger than the average actuation amplitude $d_{0}=0.1 \mathrm{~nm}$. Besides the outgoing displacement waves leaving the capillary in the axial $x$ direction, we also note the presence of circumferential displacement waves with short wavelengths around $\frac{1}{4} W$. The glycerol-like, areaaveraged actuation (3b) allows for fluctuations because it fixes only the mean vertical displacement $u_{z}$. At the resonance $f_{\text {res }}$, we find $u_{z}=(1.0 \pm 2.8) d_{0}$ on the actuation plane. We note that the standard deviation $2.8 d_{0}=0.28 \mathrm{~nm}$

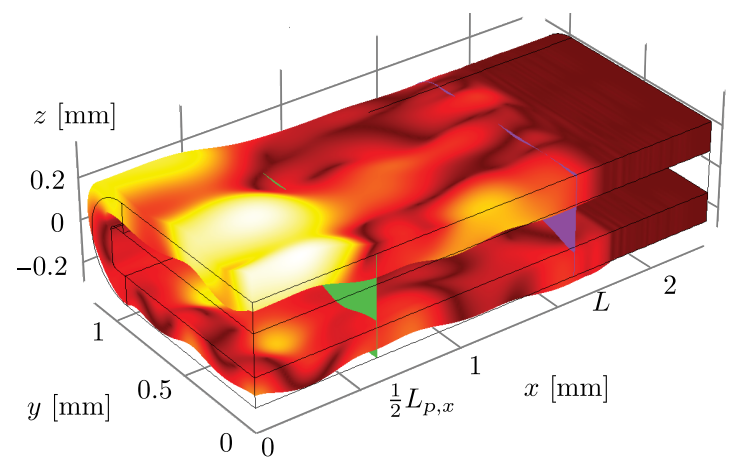

VIDEO. 2. The displacement field $\boldsymbol{u}$ in the solid (not drawn to scale) overlaid with a color plot of its magnitude $u$ from $0 \mathrm{~nm}$ (dark red) to $1.2 \mathrm{~nm}$ (white) for capillary $C 1$ at the levitating resonance $f_{\text {res }}=3.906 \mathrm{MHz}$, as in Video 1 . is very small compared to a typical coupling-layer thickness of around $0.1 \mathrm{~mm}$.

Despite the absence of a geometrical cavity along the capillary, we nevertheless find that a localized SBAW can exist in the region above the transducer. This SBAW is supported by a dynamically defined cavity that arises at resonance because the displacement of the confining glass walls becomes an order of magnitude larger in the actuation region than elsewhere. Because of its dynamic nature, the cavity is leaky: The waves in the axial direction away from the actuator region are not evanescent, but they appear as TBAWs. The presence of the TBAWs reduces the $Q$ value of the resonance state from previously reported values of $200-500$ [41] to approximately 50, a value that, however, is large enough to ensure a well-defined resonance. While combinations of standing and traveling waves have been studied for surface-acoustic-wave systems [52-55], it is remarkable that they appear here in a study of bulkacoustic-wave (BAW) systems.

To characterize the trapping capabilities of the device, we study the radiation force $\boldsymbol{F}^{\mathrm{rad}}$, Eq. (6a), acting on a $12-\mu$ m-diameter polystyrene (ps) test particle suspended in the fluid above the transducer. The $z$ component $F_{z}^{\mathrm{rad}}$ of $\boldsymbol{F}^{\mathrm{rad}}$, derived from the nearly perfect standing half wave in the vertical direction, levitates the test particle and holds it close to the pressure nodal plane. In the actuation region, this plane is nearly horizontal, with a vertical position between $-0.1 \mathrm{H}$ and 0 . Away from the actuation region, it gradually changes its vertical position and fades away, see Video 3. The maximum levitating force on the test particle

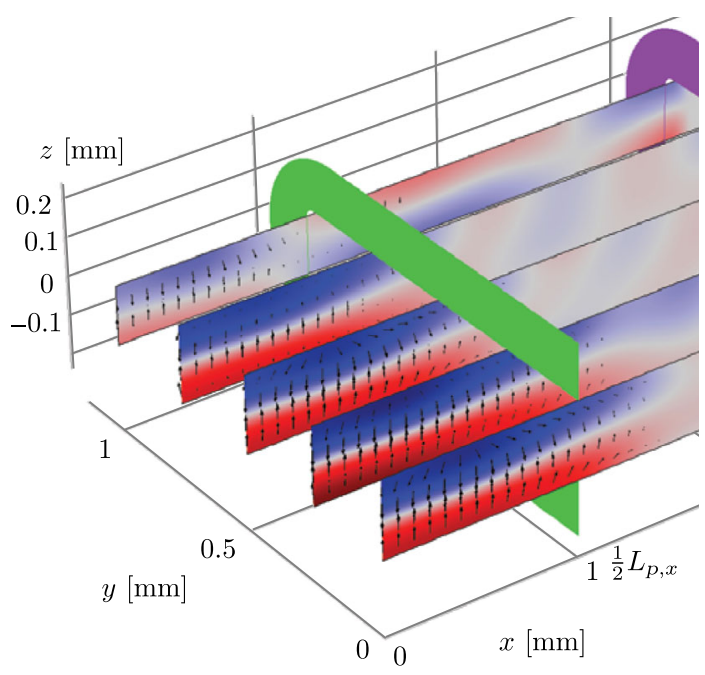

VIDEO. 3. Capillary $C 1$, color plot of the pressure field $p$ from $-0.18 \mathrm{MPa}$ (blue) to $0.18 \mathrm{MPa}$ (red) in vertical planes placed equidistantly in steps of $\frac{1}{8} W$ starting at $y=0$, showing the nearly horizontal nodal plane (gray) wobbling around $z=-0.05 \mathrm{H}$ at resonance $f_{\text {res }}=3.906 \mathrm{MHz}$. The acoustic radiation force $\boldsymbol{F}^{\mathrm{rad}}$ on $12-\mu \mathrm{m}$-diameter polystyrene test particles (black arrows with logarithmic lengths are used for visual clarity) has a maximum magnitude of $22 \mathrm{pN}$. 


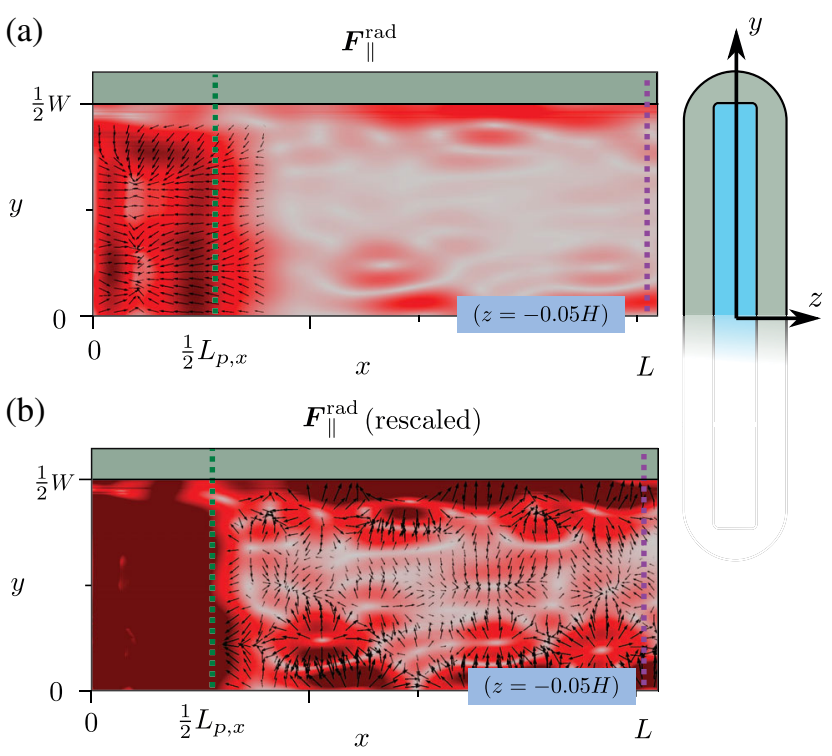

FIG. 7. Capillary $C 1$. (a) The lateral acoustic radiation force $\boldsymbol{F}_{\|}^{\mathrm{rad}}$ (the black arrows with logarithmic length) and its magnitude [the color plot from $0 \mathrm{pN}$ (gray) to $0.44 \mathrm{pN}$ (dark red)] acting on $12-\mu$ m-diameter polystyrene beads in the $x-y$ plane at $z=$ $-0.05 \mathrm{H}$ at resonance $f_{\text {res }}=3.906 \mathrm{MHz}$. (Inset) Cross-section geometry with $W=2 \mathrm{~mm}$. (b) Same as (a), but with its maximum color (dark red) decreased to $0.10 \mathrm{pN}$ to emphasize the secondary trapping points.

is $F_{z}^{\mathrm{rad}}=22 \mathrm{pN}$, which is nearly 50 times larger than the buoyancy-corrected gravitational force $F^{\text {grv }}=0.44 \mathrm{pN}$.

The lateral trapping force $\boldsymbol{F}_{\|}^{\text {rad }}$ is given by the $x$ and $y$ components $F_{x}^{\mathrm{rad}}$ and $F_{y}^{\mathrm{rad}}$ of Eq. (6a), and it acts as a retention force when a fluid flow is imposed. In Fig. 7(a) for the $C 1$ capillary at resonance $f_{\text {res }}, \boldsymbol{F}_{\|}^{\text {rad }}$ is plotted in the plane $z=$ $-0.05 H$ (the vector plot) together with its magnitude $F_{\|}^{\text {rad }}$ (the color plot). We see two clear trapping points within the actuation region, which are surrounded by strong gradients in the $x$ and $y$ directions, thus enabling lateral particle trapping. The maximum value $F_{x}^{\text {rad }}$ within the actuation region is of particular interest because it serves as a measure for the overall trapping strength of the device. We find $F_{x}^{\mathrm{rad}}=$ $0.44 \mathrm{pN}$ at the position $(x, y, z)=\left(0.3 L_{p, x}, 0,-0.05 H\right)$. Outside the actuation region, a number of weaker, secondary trapping points are observed, as shown in Fig. 7(b). Their presence is emphasized by rescaling the color range, and we note that their positions appear to be random. This finding is in qualitative agreement with experimental observations under stop-flow conditions [56].

We also verify the capability of the device to trap particles by simulating the trajectories of test particles initially placed on a regular grid throughout the trapping region and ending up in the trapping points (not shown).

Finally, we comment on the calibration of the acoustic energy density $E_{\mathrm{ac}}^{\mathrm{fl}}$ in the fluid, which controls the magnitude of the trapping force in the capillary device. Because, in practice, it is not possible to calculate $E_{\mathrm{ac}}^{\mathrm{fl}}$ in a capillary device for a known ac voltage applied to the piezoelectric transducer, it is important to measure it in any given experiment. The first method is the drop-voltage method [29], where, at first, $E_{\mathrm{ac}}^{\mathrm{fl}}$ is set high enough to levitate a test particle. Then the ac voltage, and thus $E_{\mathrm{ac}}^{\mathrm{fl}}$, is lowered until the particle drops out of the trap, at which point $F_{z}^{\mathrm{rad}}\left(E_{\mathrm{ac}}^{\mathrm{fl}}\right)=$ $\frac{4}{3} \pi a^{3}\left(\rho_{\mathrm{pa}}-\rho_{\mathrm{fl}}\right) g$ and $E_{\mathrm{ac}}^{\mathrm{fl}}$ can be found.

The second method is to increase the flow rate, and thus the flow speed $v_{\text {flow }}$, through the device until it reaches critical magnitude, where the maximum lateral retention force $F_{\| \max }^{\mathrm{rad}}$ cannot balance the Stokes drag force on the particle any longer. From a scaling argument, and introducing a coefficient $\alpha$ dependent on the device composition and geometry, we can write

$$
F_{\| \max }^{\mathrm{rad}}=\alpha \frac{4 \pi}{3} a^{3} E_{\mathrm{ac}}^{\mathrm{fl}} \frac{\omega}{c_{\mathrm{fl}}}, \quad \alpha=0.0139,
$$

where the value for $\alpha$ is found by simulating the $C 1$ capillary. The flow speed at which the Stokes drag exactly balances $F_{\| \max }^{\mathrm{rad}}$ is denoted $v_{\max }^{\text {flow }}$, so we can write

$$
F_{\| \max }^{\mathrm{rad}}=6 \pi \eta_{\mathrm{fl}} a \chi v_{\max }^{\mathrm{flow}}, \quad \chi=\chi_{\text {paral }}^{z=0}=1.064,
$$

where $\chi$ is the wall-induced drag enhancement in the center plane of a parallel-plate channel [57], and the value is for the $C 1$ capillary and the given test particle. Approximating the fluid channel to be rectangular, the maximum flow velocity can be related to the maximum flow rate $Q_{\max }^{\text {flow }}$ as $v_{\max }^{\text {flow }}=[\beta /(H W)] Q_{\max }^{\text {flow }}$, where $\beta$ is an aspect-ratiodependent constant [58], which, for $C 1$, is $\beta=1.74$. Combining Eqs. (9) and (10) leads us to

$$
E_{\mathrm{ac}}^{\mathrm{fl}}=\frac{9 \beta \chi}{2 \alpha} \frac{\eta_{\mathrm{fl}} c_{\mathrm{fl}} Q_{\mathrm{max}}^{\mathrm{flow}}}{\omega a^{2} H W} .
$$

With the assumed actuation amplitude $d_{0}=0.1 \mathrm{~nm}$, we find the maximum acoustic energy density to be $1.60 \mathrm{~J} / \mathrm{m}^{3}$, which yields the maximum flow speed and rate of $v_{\max }^{\text {flow }}=$ $3.1 \mu \mathrm{m} / \mathrm{s}$ and $Q_{\max }^{\text {flow }}=0.043 \mu \mathrm{l} / \mathrm{min}$. We emphasize that this calibration method works only if the in-plane streaming velocity is smaller than the flow velocity.

\section{B. Analysis of capillary $C 2$}

In the experiment, capillary $C 2$ is held in place by two rubber sleeves [29], which may justify a generic study using the absorbing PML region. Capillary $C 2$ differs from capillary $C 1$ by being more flat, having a cross-section aspect ratio of $1: 20$, in contrast to $1: 10$ for the latter [8]. A more significant difference is that the transducer of $C 2$ is attached by glue and not by glycerol, as with $C 1$. Therefore, we study the differing responses arising from using these two actuation conditions. 
Using the glycerol-like area-averaged actuation condition (3b), a strong levitating half-wave resonance resembling the one in $C 1$ and having a quality factor $Q=78$, is found at $f_{\text {res }}^{\text {avg }}=2.495 \mathrm{MHz}$, equal to the simple half-wave value $c_{\mathrm{fl}} /(2 H)$. The maximum levitating force is $F_{z}^{\mathrm{rad}}=18 \mathrm{pN}$.

Turning to the gluelike rigid actuation condition (3a), the resonance frequency drops slightly to $f_{\text {res }}^{\text {rgd }}=2.345 \mathrm{MHz}$, fairly close to the experimentally observed resonance at $2.585 \mathrm{MHz}$, while the quality factor decreases to $Q=61$, the acoustic energy density increases by $9 \%$, and the maximum levitating force is nearly doubled to become $F_{z}^{\mathrm{rad}}=33 \mathrm{pN}$. Qualitatively, the frequency spectrum changes from having an additional resonance peak close to the levitating resonance of $f_{\text {res }}=2.495 \mathrm{MHz}$ using the glycerol-like condition to having no extra resonances using the gluelike condition. The spatial structure of the resonance field in the fluid at $f_{\text {res }}$ largely remains the same for the two different boundary conditions.

\section{Analysis of capillary $\mathbf{C 3}$}

For the quadratic capillary $C 3$ [27], we use the nominal geometry parameters $W=H=100 \mu \mathrm{m}$ and $H_{\mathrm{gl}}=50 \mu \mathrm{m}$ with PML absorption, and we predict numerically the frequency of the levitating half-wave resonance to be $f_{\text {res }}=7.35 \mathrm{MHz}$, only $2 \%$ from the simple half-wave value $c_{\mathrm{fl}} /(2 \mathrm{H})=7.49 \mathrm{MHz}$, and $7 \%$ lower than the experimental value, $f_{\text {res }}^{\exp }=7.90 \mathrm{MHz}$. This discrepancy leads us to study changes in the predicted acoustic response as a function of geometrical variations within the fabrication uncertainties listed by the producer (VitroCom No. 8510, Mountain Lakes, New Jersey): $\pm 10 \%$ for $W$ and $H$, and $\pm 20 \%$ for $H_{\mathrm{gl}}$. Some of the resulting numerical predictions for the resonance frequency $f_{\text {res }}$ and $Q$ values, using both the averaged and rigid actuation condition, are listed in Table V.

For these thin-wall capillaries, the levitating half-wave resonance depends strongly on the channel height $H$ and

TABLE V. Simulation results for the quadratic capillary $C 3$ : the levitating half-wave resonance frequency $f_{\text {res }}$, the quality factor $Q$, and the acoustic energy density $E_{\mathrm{ac}}^{\mathrm{fl}}$. The side length $W=H$ and the glass thickness $H_{\mathrm{gl}}$ are varied within the fabrication specifications, and we use either the averaged actuation condition $A$, Eq. (3b), or the rigid condition $R$, Eq. (3a). The experimental resonance frequency is $f_{\text {res }}^{\exp }=7.90 \mathrm{MHz}$.

\begin{tabular}{lcccrr}
\hline \hline$W=H[\mu \mathrm{m}]$ & $H_{\mathrm{gl}}[\mu \mathrm{m}]$ & Actuation & $f_{\text {res }}[\mathrm{MHz}]$ & $Q$ & $E_{\mathrm{ac}}^{\mathrm{fl}}[\mathrm{Pa}]$ \\
\hline 90 & 40 & $A$ & 8.230 & 114 & 67 \\
90 & 60 & $A$ & 7.964 & 60 & 28 \\
90 & 60 & $R$ & 7.950 & 117 & 163 \\
100 & 50 & $A$ & 7.350 & 91 & 40 \\
100 & 50 & $R$ & 7.313 & 157 & 120 \\
110 & 40 & $A$ & 6.670 & 124 & 26 \\
110 & 40 & $R$ & 6.640 & 148 & 84 \\
110 & 60 & $A$ & 6.590 & 79 & 28 \\
\hline \hline
\end{tabular}

width $W$, and much less on the glass thickness $H_{\mathrm{gl}}$. The $10 \%$ variation $H=W=(100 \pm 10) \mu \mathrm{m}$ leads to a $10 \%$ variation in $f_{\text {res }} \approx(7.4 \pm 0.7) \mathrm{MHz}$ for a fixed $H_{\mathrm{gl}}$, while the $20 \%$ variation in $H_{\mathrm{gl}}=(50 \pm 10) \mu \mathrm{m}$ leads to variations of less than $2 \%$ in $f_{\text {res }}$ for a fixed $H$ and $W$. Moreover, in agreement with the results for $C 2, E_{\mathrm{ac}}^{\mathrm{fl}}$ and the $Q$ factor for the gluelike rigid actuation condition $R$ are increased by factors of 3-6 and 1.2-3, respectively, compared to those of the glycerol-like averaged condition $A$. The maximum levitating force with $H=W=100 \mu \mathrm{m}$ for condition $R$ is $F_{z}^{\mathrm{rad}}=3690 \mathrm{pN}$, while it drops to $1380 \mathrm{pN}$ for condition $A$. The two actuation types lead to the same levitating resonance frequency within $0.5 \%$.

\section{Analysis of capillary $\boldsymbol{C 4}$}

In the original work on the circular capillary $C 4$ [28], effects due to reflections at the end of the capillary were discussed. Therefore, we study numerically the frequency response with PML absorption (perfect absorption of all outgoing waves) and without it (reflection of all outgoing waves at the end wall without absorption).

For the case with PML absorption, we find the levitating half-wave resonance to have the following characteristics: $f_{\text {res }}=0.9753 \mathrm{MHz}, Q=109, E_{\mathrm{ac}}^{\mathrm{fl}}=0.96 \mathrm{~Pa}$, and maximum levitating force $F_{z}^{\mathrm{rad}}=1.6 \mathrm{pN}$ using the glycerollike condition (3b), and $f_{\text {res }}=0.9818 \mathrm{MHz}, Q=79$, $E_{\mathrm{ac}}^{\mathrm{fl}}=0.57 \mathrm{~Pa}$, and the maximum levitating force $F_{z}^{\mathrm{rad}}=$ $2.6 \mathrm{pN}$ using the gluelike condition (3a). These values for $f_{\text {res }}$ are close to the experimental value of $0.981 \mathrm{MHz}$ and the theoretical value $\gamma_{11} c_{\mathrm{fl}} /(\pi H)=1.03 \mathrm{MHz}$ based on the simple hard-wall cylinder geometry, where $\gamma_{11}=1.841$ is the first zero of the derivative $J_{1}^{\prime}(x)$ of the first Bessel function. As for the other capillaries, the choice of actuation condition leads to no qualitative-and only minor quantitative-differences in the frequency spectrum.

When the PML is removed by imposing the zero-stress condition at $x=L$, the outgoing waves from the transducer region are reflected by the end wall, and complex interference effects arise in the capillary. For a fixed $L_{p, x}$, the channel length $L$ is varied between 1 and $10 \mathrm{~mm}$, and, relative to the PML case, we find that the levitating resonance frequency changes only about $1 \%$, while the $Q$ value and $E_{\mathrm{ac}}^{\mathrm{fl}}$ typically increase by $200 \%-300 \%$ and $400 \%-600 \%$, respectively. This result illustrates that whether the capillary ends are reflecting or fully absorbing does not affect the frequency of the main levitating mode significantly, while it has a crucial influence on the $Q$ factors and acoustic energies, as well as on the existence of secondary resonance modes. By contrast, the internal attenuation, given by the $\Gamma$ factors, is unimportant since the associated attenuation lengths $L_{\mathrm{fl}}=\left[\left(c_{\mathrm{fl}}\right) / \omega \Gamma_{\mathrm{fl}}\right] \approx 60 \mathrm{~mm}$ and $L_{\mathrm{sl}}=\left[\left(c_{\mathrm{sl}}\right) / \omega \Gamma_{\mathrm{sl}}\right] \approx 2 \mathrm{~m}$ are longer than the capillary half length $L \approx 20 \mathrm{~mm}$. 


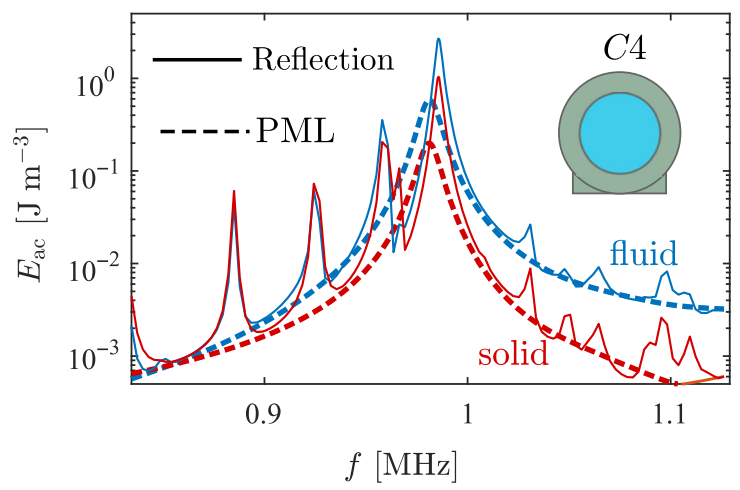

FIG. 8. Simulated frequency response of capillary $C 4$ (the inset shows its cross section), with a total length of $L=10 \mathrm{~mm}$ and using the gluelike actuation condition, Eq. (3b). Semilog plot of the volume-averaged acoustic energy density $E_{\mathrm{ac}}^{\mathrm{fl}}$ in the trapping region $\left(x<\frac{1}{2} L_{p}\right)$ for the fluid (blue) and the solid (red), both with PMLs (the thick dashed lines, $k_{\mathrm{PML}}=10^{3}$, $f_{\text {res }}^{\mathrm{PML}}=0.9812 \mathrm{MHz}$ ) and without (the solid lines, $k_{\mathrm{PML}}=$ $\left.0, f_{\text {res }}^{0}=0.9856 \mathrm{MHz}\right)$.

For the longest capillary $L=10 \mathrm{~mm}$, we plot in Fig. 8 $E_{\text {ac }}$ as a function of frequency $f$ near the levitating half-wave resonance $f_{\text {res }}$ with and without PML absorption. First, we note that $f_{\text {res }}$ in the two cases differs by less than $0.4 \%$. Second, without PML absorption, the energy is not lost by the outgoing waves and, consequently, the line shape becomes higher and more narrow. Indeed, the resonance amplitude $E_{\mathrm{ac}}^{\mathrm{fl}}$ increases from 0.58 $\mathrm{Pa}$ (PML) to $2.69 \mathrm{~Pa}$ (no PML), while the $Q$ factor increases from 109 (PML) to 252 (no PML). Third, without PML, the reflections from the end wall give rise to a number of additional, nearly equidistant, smaller resonance peaks, reminiscent of a Fabry-Pérot-like interference condition for the acoustic wave in the $x$ direction. Finally, without PML, the reflections lead to a more irregular spatial pattern of the levitating resonance mode in the actuation region (not shown) than for that of the mode with PML absorption and no reflections.

Because of the complex nature of the coupling between pressure waves in the fluid and the compressional and shear waves in the solid, we have been able to interpret $f_{\text {res }}$ only for the levitating resonance mode - not for other modesin simple terms of the three sound speeds of the system, listed in Table I, combined with the geometrical length scales of capillary $C 4$, listed in Table II.

\section{E. Analysis of capillary $C 5$}

We end our numerical modeling of the capillaries by a study of capillary $C 5$, which is a geometry defined by us and not studied in the literature. The focus here is to obtain a more regular spatial pattern of the mode with spatial variations on the longest possible length scale.

The study in Sec. III D of the circular capillary $C 4$ reveals that spatial irregularities in the levitating resonance mode are reduced when the reflections from the end wall

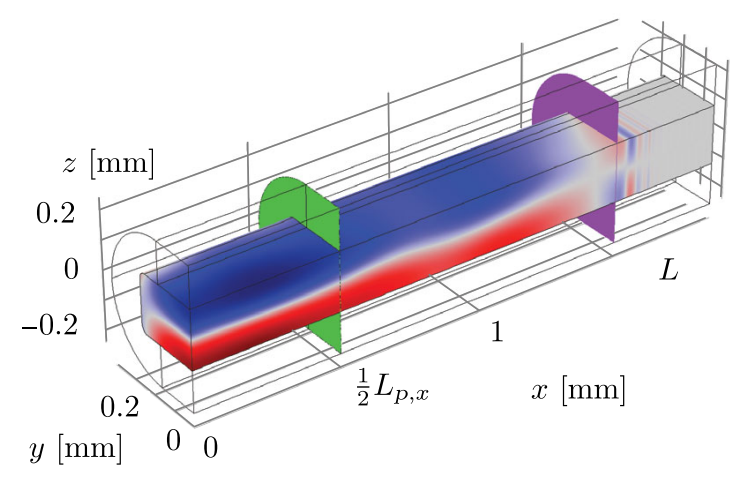

VIDEO. 4. Capillary $C 5$, color plot of the pressure field $p$ in the fluid from $-0.10 \mathrm{MPa}$ (blue) to $0.10 \mathrm{MPa}$ (red) at the resonance frequency $f_{\text {res }}=4.201 \mathrm{MHz}$. The green and purple planes represent the end of the actuation region and the beginning of the PML domain, respectively.

are absorbed by a PML. We also find this tendency in the study in Sec. III A of the wide, flat capillary $C 1$, where, even in the presence of a PML, the levitating resonance mode has significant spatial variations, see Video 2 and Fig. 7(b). These spatial variations are caused by the relative high mode numbers in the solid, and therefore we design capillary $C 5$ as a version of capillary $C 1$ with its width reduced by a factor of 4 to $W=0.5 \mathrm{~mm}$, thus reducing the cross-section aspect ratio $W / H$ from 10 to 2.5 .

The separation of resonance frequencies increases in the narrow capillary $C 5$. In the range between 3.5 and $4.4 \mathrm{MHz}$, it contains only two resonance peaks (at 3.572 and 4.201 MHz), whereas capillary $C 1$ in the same interval contains three pronounced peaks and four smaller ones. The resonance at $f_{\text {res }}=4.201 \mathrm{MHz}$, with $Q=53$, is a levitating mode well suited for acoustic trapping, and, in Videos 4 and 5, we plot the corresponding pressure field $p$ and displacement field $\boldsymbol{u}$. The spatial structure of the resonance is more regular, especially in the solid, where the displacement in the $y-z$ plane now resembles a simple fundamental mode with a pivoting motion around a single stationary point away from the symmetry plane (the black point on the $y$ axis in the inset of Video 5). Compared to

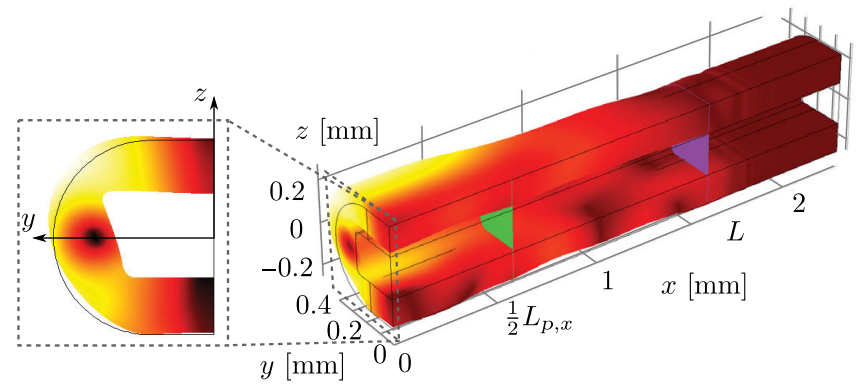

VIDEO. 5. Capillary $C 5$, the displacement field $\boldsymbol{u}$ in the solid (not drawn to scale) overlaid with a color plot of its magnitude $u$ from $0 \mathrm{~nm}$ (dark red) to $1.2 \mathrm{~nm}$ (white) at the resonance frequency $f_{\text {res }}=4.201 \mathrm{MHz}$, as in Video 3 . 


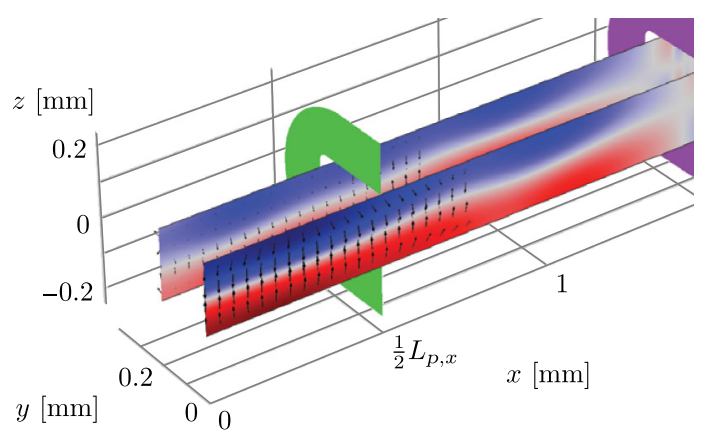

VIDEO. 6. Capillary $C 5$, a color plot of the pressure field $p$ from $-0.10 \mathrm{MPa}$ (blue) to $0.10 \mathrm{MPa}$ (red) in vertical planes placed equidistantly in steps of $\frac{3}{8} W$ starting at $y=0$ showing the nearly horizontal nodal plane (gray) near $z=-0.03 H$ at resonance $f_{\text {res }}=4.201 \mathrm{MHz}$. The acoustic radiation force $\boldsymbol{F}^{\mathrm{rad}}$ on $12-\mu$ m-diameter polystyrene test particles (black arrows with logarithmic lengths are shown for visual clarity) has a maximum magnitude of $7 \mathrm{pN}$.

that of capillary $C 1$, the levitating mode of capillary $C 5$ stretches far outside the actuation region and it is more regular in space, but its amplitude is reduced approximately by a factor of 1.8 , and the maximum levitating force drops a factor of 3 to $F_{z}^{\mathrm{rad}}=7 \mathrm{pN}$. The pressure nodal surface of the levitating mode is weakly wobbling around the horizontal plane located at $z=-0.03 H$; see Video 6. This wobbling is less pronounced than the one we find in capillary $C 1$, which, moreover, is displaced farther downward-namely, to $z=-0.05 H$.

Compared to capillary $C 1$, the reduced pressure amplitude in capillary $C 5$ leads to a corresponding reduction in the lateral radiation force by a factor of $1.8^{2}=3.2$, plotted for $z=-0.03 H$ in Fig. 9, and it has a more regular spatial structure compared to that shown in Fig. 7. The main lateral trapping points are now confined to the intersection of the nodal plane and the $x-z$ plane inside the actuation region, although the levitating mode extends far beyond the (a)

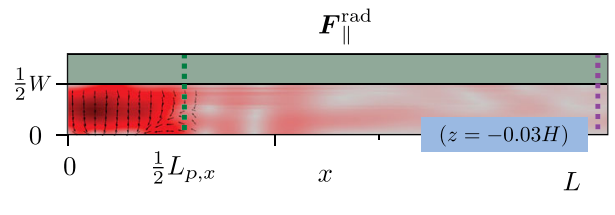

(b)

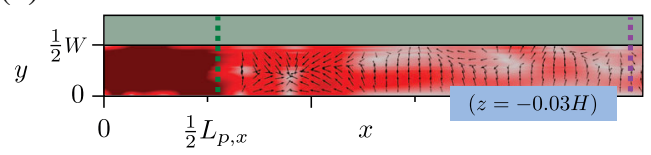

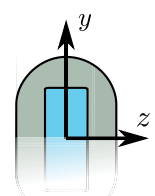

actuation region; see Video 6. There do exist secondary trapping points outside the actuation region, and compared to those of capillary $C 1$ they are relatively strong, but they are fewer in number and appear in a more regular pattern. This finding is in qualitative agreement with experimental observations on the circular capillary $C 4$ [28].

\section{DISCUSSION}

The main outcome of our numerical modeling of glasscapillary devices is that, for each of the five capillaries $C 1-C 5$ in our study, we find that a localized acoustic resonance mode, the so-called levitating mode, existseven though the completely straight capillaries do not contain any geometrical cavities in the axial $x$ direction. This levitating mode is the result of a strong vertical SBAW forming in the region $x<\frac{1}{2} L_{p}$ of the capillary directly above the transducer. Although the bottom glass wall attached to the transducer is displaced only by $d_{0}=0.1 \mathrm{~nm}$, the opposite top glass wall above the transducer is displaced an order of magnitude more, approximately $10 d_{0}$. This relatively large displacement results in a dynamically defined cavity that provides sufficient confinement for the vertical SBAW, although acoustic energy is lost by a horizontal TBAW radiating away from the cavity for $x>\frac{1}{2} L_{p}$. The levitating modes have a relatively high $Q$ value, 50 or higher, and they all exhibit good trapping characteristics: a nearly horizontal pressure nodal plane near the center of the channel (see Videos 3 and 6), with sufficiently strong lateral acoustic forces (see Figs. 7 and 9).

The values of the levitating resonance frequencies $f_{\text {res }}$ are in good agreement with the experimental values obtained for capillaries $C 1-C 4$ [8,27-29], as listed in Table II. They vary by less than $1 \%$ as we change the boundary condition at the outlet from an ideally absorbing PML region to the no-stress condition of a capillary facing air, and as we change the actuation from glycerol-like area-averaged displacement to gluelike rigid displacement. However, we find that varying these idealized generic boundary conditions does result in significant changes of the acoustic energy density $E_{\mathrm{ac}}^{\mathrm{fl}}$ and the acoustic force $\boldsymbol{F}^{\mathrm{rad}}$ on a test particle, as well as the resonance quality factor $Q$. In general, we find two opposing trends: On the one hand, the absorption of the PML region reduces both the acoustic energy and the trapping forces by a factor of 2-6, which is a drawback for achieving good trapping. On the other hand, the presence of the PML region has three beneficial aspects: It removes the sensitivity to reflections of acoustic waves at the outlet wall, it results in more regular spatial variations, and it makes the trapping strength independent of the position of the transducer relative to the capillary ends.

The submillimeter dimensions of the capillary cross section implies that the levitating resonance frequency $f_{\text {res }}$ is sensitive to the exact geometry. We find that, when changing the geometry within the tolerances listed by the manufacturer of the capillaries, the value of the levitating 
resonance can vary by as much as $10 \%$, or by up to $0.7 \mathrm{MHz}$ for a 7-MHz resonance. Because the levitating resonance is close to an ideal standing half-wave resonance in the channel of height $H$, we have $f_{\text {res }} \propto H^{-1}$, and the most critical length parameter therefore becomes $H$.

Turning to the acoustic radiation force acting on a $12-\mu \mathrm{m}$-diameter polystyrene test particle in the device, our model correctly predicts that this force is present in the actuation region with a magnitude large enough to support one or more stable trapping points there. For the assumed actuation amplitude $d_{0}=0.1 \mathrm{~nm}$, the maximum levitating force $F^{\text {rad }}$ ranges from $2 \mathrm{pN}$ (the circular capillary $C 4$ ) to $3690 \mathrm{pN}$ (the square capillary $C 3$ ), which is between 5 and 8000 times the buoyancy-corrected gravitational force $F^{\text {grv }}$, depending, of course, on the transducer length $L_{p, x}$. Our model also correctly predicts the existence of weaker, secondary trapping points in the region outside the actuation region, see Figs. 7(b) and 9(b), in agreement with published experiments $[28,56]$. However, even without the PML absorption, the magnitude of the lateral acoustic force $\boldsymbol{F}_{\|}^{\mathrm{rad}}$ is small for most geometries, $F_{\|}^{\mathrm{rad}} / F^{\mathrm{grv}} \approx 1-10$, the exception being capillary $C 3$ with $F_{\|}^{\mathrm{rad}} / F^{\text {grv }}>100$. This small magnitude indicates that the performance of actual acoustic capillary traps may depend on additional forces such as streaming-induced drag forces and particle-particle interactions. For idealized systems with a rectangular cross section and translation invariance in the axial $x$ direction, the critical particle radius $a_{c}$, below which streaming drag dominates, can be estimated by $a_{c}=\delta \sqrt{3 \Psi / \Phi}$ [36], where $\delta$ is the boundary-layer thickness, $\Psi$ is the streaming coefficient ( $=\frac{3}{8}$ for a planar wall), and $\Phi$ is the acoustic contrast factor characterizing the strength of the acoustic radiation force. For the levitating mode in $3 \mathrm{D}$, the lateral component of the radiation force is smaller by a factor of $\alpha$ than the levitating vertical one [see Eq. (9)], so, in this case, we estimate $a_{c}=\delta \sqrt{3 \Psi /(\alpha \Phi)}$. Numerically, we find $\alpha \approx 0.01$, see Eq. (9), and this relative weakening of the lateral radiation force indicates that the critical particle radius may be 1 order of magnitude larger than the $1 \mu \mathrm{m}$ found for typical BAW devices [36,43].

While a wide capillary is good for enhanced throughput, it suffers from the existence of high-mode, short-wavelength elastic waves propagating around the perimeter of the capillary. We find that reducing the aspect ratio removes these perimeter waves while maintaining the good characteristics of the levitating mode. Other irregular spatial patterns in the levitating mode and the acoustic-trapping force are induced by reflections of acoustic waves at the end of the capillary. We find that, by implementing an ideally absorbing PML region, these reflections are removed, and a more regular spatial behavior of the trap is obtained. While the reduction in aspect ratio reduces the throughput and the introduction of absorption reduces the trapping force by a factor of $2-6$, the resulting increased regularity in spatial behavior, the increased separation of modes in frequency implying reduced mixing of unwanted modes, and the decreased sensitivity to the exact location of the transducer relative to the capillary ends may be beneficial design considerations worth taking into account. The redesigned devices may be more robust to small perturbations in the device geometry and mechanical actuation.

Our numerical modeling of the capillary systems for acoustic trapping is in fair agreement with the existing experimental data in the literature. However, to fully establish its capability as a design tool for optimizing acoustic trapping, more experimental data is needed to characterize the acoustic properties more fully. Here, stopflow experiments as in Ref. [56] would be helpful, as would more systematic reporting of the acoustic energy density present in the devices using, say, the drop-voltage method [29] or the flow versus retention force briefly mentioned in Sec. III A. Also, systematic reporting of the magnitude of the acoustic streaming would be helpful because there might be situations in the capillary systems, where the acoustic streaming is so large that it is comparable to the flow velocities in the channel. If this were the case, our analysis of the lateral acoustic forces must be supplemented by a discussion of the drag forces from acoustic streaming.

\section{CONCLUSION}

The increased use of acoustic-trapping devices in microfluidic handling of particles allows for the development of alternative functionalities in contemporary biotechnology. In this paper, we develop a three-dimensional numerical model for water-filled glass capillaries, which may be a useful design tool for the future development of acoustic microparticle traps. We validate the model and demonstrate how it captures many of the experimental observations reported in the literature for four different glass-capillary devices, particularly regarding the frequency, the $Q$ factor, as well as the spatial structure and trapping capability of levitating resonance modes. Our model explains the physical mechanism leading to these localized resonance modes in terms of a dynamically defined cavity in the region above the transducer.

We demonstrate the potential of the model as a design tool through an analysis of the sensitivity of the system to changes in the geometry, the specific actuation condition, and the acoustic absorption. We find that, while the existence of a main levitating resonance mode and the value of the resonance frequency are relatively insensitive to these changes, the opposite is true for the magnitude of the trapping force and the lateral spatial structure: The trapping force is strongly increased for rectangular cross sections with a low aspect ratio-the best being the square shape-and the lateral spatial structure of the levitating mode becomes more uniform. For the case without acoustic absorption, reflections at the outlet of the capillary make these responses very sensitive to the length 
of the system. The introduction of deliberate absorption of acoustic waves at the outlet would lower this sensitivity and would probably result in more-robust devices. However, this benefit in device operation must be weighed against a decreased magnitude of the acoustic forces.

Our numerical model takes many, but certainly not all, physical relevant aspects into account. The obvious next step, which we are currently working on, is to include the drag force from acoustic streaming, as well as both acoustic and hydrodynamic particle-particle interaction effects. These more elaborate phenomena all involve lateral forces comparable to, or possibly even larger than, the lateral single-particle acoustic radiation force $\boldsymbol{F}_{\|}^{\mathrm{rad}}$ calculated in our model. The simulation of these phenomena relies on numerically well-characterized acoustic fields, which, we believe, are provided by the model we present here. Possible model strategies to pursue in such calculations were outlined by Muller and Bruus [32], Muller et al. [36] and Lei et al. [59] for the case of acoustic streaming, by Silva and Bruus [60] for acoustic particle-particle interactions, and by Ley and Bruus [61] for hydrodynamic particle-particle interactions.

Our numerical model reveals that the acoustic-trapping devices based on simple glass capillaries are perhaps not as simple as they appear at first glance.

\section{ACKNOWLEDGMENTS}

We thank our collaborators Carl Johannsson, Mikael Evander, and Thomas Laurell, from Lund University in Sweden, for the valuable discussions related to experimental aspects. This work was supported by Lund University and the Knut and Alice Wallenberg Foundation (Grant No. KAW 2012.0023).

[1] H. Bruus, J. Dual, J. Hawkes, M. Hill, T. Laurell, J. Nilsson, S. Radel, S. Sadhal, and M. Wiklund, Forthcoming Lab on a Chip tutorial series on acoustofluidics: Acoustofluidicsexploiting ultrasonic standing wave forces and acoustic streaming in microfluidic systems for cell and particle manipulation, Lab Chip 11, 3579 (2011).

[2] Microscale Acoustofluidics, edited by T. Laurell and A. Lenshof (Royal Society of Chemistry, Cambridge, England, 2015).

[3] P. Thevoz, J. D. Adams, H. Shea, H. Bruus, and H. T. Soh, Acoustophoretic synchronization of mammalian cells in microchannels, Anal. Chem. 82, 3094 (2010).

[4] P. Augustsson, C. Magnusson, M. Nordin, H. Lilja, and T. Laurell, Microfluidic, label-free enrichment of prostate cancer cells in blood based on acoustophoresis, Anal. Chem. 84, 7954 (2012).

[5] X. Ding, S.-C. S. Lin, B. Kiraly, H. Yue, S. Li, I.-K. Chiang, J. Shi, S. J. Benkovic, and T. J. Huang, On-chip manipulation of single microparticles, cells, and organisms using surface acoustic waves, Proc. Natl. Acad. Sci. U.S.A. 109, 11105 (2012).

[6] D. J. Collins, B. Morahan, J. Garcia-Bustos, C. Doerig, M. Plebanski, and A. Neild, Two-dimensional single-cell patterning with one cell per well driven by surface acoustic waves, Nat. Commun. 6, 8686 (2015).

[7] B. Hammarström, M. Evander, H. Barbeau, M. Bruzelius, J. Larsson, T. Laurell, and J. Nillsson, Non-contact acoustic cell trapping in disposable glass capillaries, Lab Chip 10, 2251 (2010).

[8] B. Hammarström, T. Laurell, and J. Nilsson, Seed particle enabled acoustic trapping of bacteria and nanoparticles in continuous flow systems, Lab Chip 12, 4296 (2012).

[9] B. Hammarström, M. Evander, J. Wahlström, and J. Nilsson, Frequency tracking in acoustic trapping for improved performance stability and system surveillance, Lab Chip 14, 1005 (2014).

[10] B. W. Drinkwater, Dynamic-field devices for the ultrasonic manipulation of microparticles, Lab Chip 16, 2360 (2016).

[11] D. J. Collins, C. Devendran, Z. Ma, J. W. Ng, A. Neild, and Y. Ai, Acoustic tweezers via sub-time-of-flight regime surface acoustic waves, Sci. Adv. 2, e1600089 (2016).

[12] H. G. Lim, Y. Li, M.-Y. Lin, C. Yoon, C. Lee, H. Jung, R. H. Chow, and K. K. Shung, Calibration of trapping force on cell-size objects from ultrahigh-frequency single-beam acoustic tweezer, IEEE Trans. Ultrason. Ferroelectr. Freq. Control 63, 1988 (2016).

[13] D. Baresch, J.-L. Thomas, and R. Marchiano, Observation of a Single-Beam Gradient Force Acoustical Trap for Elastic Particles: Acoustical Tweezers, Phys. Rev. Lett. 116, 024301 (2016).

[14] A. Riaud, M. Baudoin, O. Bou Matar, L. Becerra, and J.-L. Thomas, Selective Manipulation of Microscopic Particles with Precursor Swirling Rayleigh Waves, Phys. Rev. Applied 7, 024007 (2017).

[15] J. Zhang, S. Yan, D. Yuan, G. Alici, N.-T. Nguyen, M. E. Warkiani, and W. Li, Fundamentals and applications of inertial microfluidics: A review, Lab Chip 16, 10 (2016).

[16] J. Voldman, Electrical forces for microscale cell manipulation, Annu. Rev. Biomed. Eng. 8, 425 (2006).

[17] D. Grier, A revolution in optical manipulation, Nature (London) 424, 810 (2003).

[18] J. Nilsson, M. Evander, B. Hammarström, and T. Laurell, Review of cell and particle trapping in microfluidic systems, Anal. Chim. Acta 649, 141 (2009).

[19] B. Cetin, M. B. Ozer, and M. E. Solmaz, Microfluidic bio-particle manipulation for biotechnology, Biochem. Eng. J. 92, 63 (2014).

[20] J. T. Karlsen and H. Bruus, Forces acting on a small particle in an acoustical field in a thermoviscous fluid, Phys. Rev. E 92, 043010 (2015).

[21] M. Wiklund, Acoustofluidics 12: Biocompatibility and cell viability in microfluidic acoustic resonators, Lab Chip 12, 2018 (2012).

[22] M. Grundy, W. Bolek, W. Coakley, and E. Benes, Rapid agglutination testing in an ultrasonic standing wave, J. Immunol. Methods 165, 47 (1993).

[23] P. Vainshtein, M. Fichman, K. Shuster, and C. Gutfinger, The effect of centreline particle concentration in a wave tube, J. Fluid Mech. 306, 31 (1996). 
[24] M. Wiklund, S. Nilsson, and H. Hertz, Ultrasonic trapping in capillaries for trace-amount biomedical analysis, J. Appl. Phys. 90, 421 (2001).

[25] D. N. Ankrett, D. Carugo, J. Lei, P. Glynne-Jones, P. A. Townsend, X. Zhang, and M. Hill, The effect of ultrasound-related stimuli on cell viability in microfluidic channels, J. Nanobiotechnol. 11, 20 (2013).

[26] D. Carugo, D. N. Ankrett, P. Glynne-Jones, L. Capretto, R. J. Boltryk, X. Zhang, P. A. Townsend, and M. Hill, Contrast agent-free sonoporation: The use of an ultrasonic standing wave microfluidic system for the delivery of pharmaceutical agents, Biomicrofluidics 5, 044108 (2011).

[27] P. Mishra, M. Hill, and P. Glynne-Jones, Deformation of red blood cells using acoustic radiation forces, Biomicrofluidics 8, 034109 (2014).

[28] I. Gralinski, S. Raymond, T. Alan, and A. Neild, Continuous flow ultrasonic particle trapping in a glass capillary, J. Appl. Phys. 115, 054505 (2014).

[29] J. Lei, P. Glynne-Jones, and M. Hill, Acoustic streaming in the transducer plane in ultrasonic particle manipulation devices, Lab Chip 13, 2133 (2013).

[30] M. Evander, O. Gidlöf, B. Olde, D. Erlinge, and T. Laurell, Non-contact acoustic capture of microparticles from small plasma volumes, Lab Chip 15, 2588 (2015).

[31] P. Ohlsson, M. Evander, K. Petersson, L. Mellhammar, A. Lehmusvuori, U. Karhunen, M. Soikkeli, T. Seppa, E. Tuunainen, A. Spangar, P. von Lode, K. Rantakokko-Jalava, G. Otto, S. Scheding, T. Soukka, S. Wittfooth, and T. Laurell, Integrated acoustic separation, enrichment, and microchip polymerase chain reaction detection of bacteria from blood for rapid sepsis diagnostics, Anal. Chem. 88, 9403 (2016).

[32] P. B. Muller and H. Bruus, Numerical study of thermoviscous effects in ultrasound-induced acoustic streaming in microchannels, Phys. Rev. E 90, 043016 (2014).

[33] P. Hahn and J. Dual, A numerically efficient damping model for acoustic resonances in microfluidic cavities, Phys. Fluids 27, 062005 (2015).

[34] Corning Inc., "Glass silicon constraint substrates" http:// www.valleydesign.com/Datasheets/Corning\%20Pyrex $\% 207740$.pdf.

[35] CRC Handbook of Chemistry and Physics, 95th ed., edited by W. M. Haynes (CRC Press, Boca Raton, FL, 2014).

[36] P. B. Muller, R. Barnkob, M. J. H. Jensen, and H. Bruus, A numerical study of microparticle acoustophoresis driven by acoustic radiation forces and streaming-induced drag forces, Lab Chip 12, 4617 (2012).

[37] P. H. Mott, J. R. Dorgan, and C. M. Roland, The bulk modulus and Poisson's ratio of "incompressible" materials, J. Sound Vib. 312, 572 (2008).

[38] L. Bergmann, Der Ultraschall und seine Anwendung in Wissenschaft und Technik, 6th ed. (S. Hirzel Verlag, Stuttgart, 1954).

[39] P. B. Muller and H. Bruus, Theoretical study of timedependent, ultrasound-induced acoustic streaming in microchannels, Phys. Rev. E 92, 063018 (2015).

[40] F. Garofalo, T. Laurell, and H. Bruus, Performance Study of Acoustophoretic Microfluidic Silicon-Glass Devices by Characterization of Material- and Geometry-Dependent Frequency Spectra, Phys. Rev. Applied 7, 054026 (2017).
[41] R. Barnkob, P. Augustsson, T. Laurell, and H. Bruus, Measuring the local pressure amplitude in microchannel acoustophoresis, Lab Chip 10, 563 (2010).

[42] P. Augustsson, R. Barnkob, S. T. Wereley, H. Bruus, and T. Laurell, Automated and temperature-controlled micro-PIV measurements enabling long-term-stable microchannel acoustophoresis characterization, Lab Chip 11, 4152 (2011).

[43] P. B. Muller, M. Rossi, A. G. Marin, R. Barnkob, P. Augustsson, T. Laurell, C. J. Kähler, and H. Bruus, Ultrasound-induced acoustophoretic motion of microparticles in three dimensions, Phys. Rev. E 88, 023006 (2013).

[44] F. Collino and P. B. Monk, Optimizing the perfectly matched layer, Comput. Methods Appl. Mech. Eng. 164, 157 (1998).

[45] A. D. Pierce, Acoustics (Acoustical Society of America, Woodbury, NY, 1991).

[46] L. D. Landau and E. M. Lifshitz, Theory of Elasticity, 3rd ed., Course of Theoretical Physics Vol. 7 (Pergamon Press, Oxford, 1986).

[47] M. Settnes and H. Bruus, Forces acting on a small particle in an acoustical field in a viscous fluid, Phys. Rev. E 85, 016327 (2012).

[48] M. M. Gregersen, M. B. Andersen, G. Soni, C. Meinhart, and H. Bruus, Numerical analysis of finite Debye-length effects in induced-charge electro-osmosis, Phys. Rev. E 79, 066316 (2009).

[49] COMSOL MULTIPHYSICS 5.2a, http://www.comsol.com (2016).

[50] A. F. Oskooi, L. Zhang, Y. Avniel, and S. G. Johnson, The failure of perfectly matched layers, and towards their redemption by adiabatic absorbers, Opt. Express 16, 11376 (2008).

[51] See Supplemental Material at http://link.aps.org/ supplemental/10.1103/PhysRevApplied.8.024020 for animated GIF versions of Videos 1-6.

[52] N. Nama, R. Barnkob, Z. Mao, C. J. Kähler, F. Costanzo, and T. J. Huang, Numerical study of acoustophoretic motion of particles in a PDMS microchannel driven by surface acoustic waves, Lab Chip 15, 2700 (2015).

[53] C. Devendran, N. R. Gunasekara, D. J. Collins, and A. Neild, Batch process particle separation using surface acoustic waves (SAW): Integration of travelling and standing SAW, RSC Adv. 6, 5856 (2016).

[54] C. Devendran, T. Albrecht, J. Brenker, T. Alan, and A. Neild, The importance of travelling wave components in standing surface acoustic wave (SSAW) systems, Lab Chip 16, 3756 (2016).

[55] J. W. Ng, D. J. Collins, C. Devendran, Y. Ai, and A. Neild, Flow-rate-insensitive deterministic particle sorting using a combination of travelling and standing surface acoustic waves, Microfluid. Nanofluid. 20, 151 (2016).

[56] M. W. H. Ley, C. Johannesson, M. Evander, T. Laurell, and H. Bruus, in Proceedings of the 20th International Conference on Miniaturized Systems for Chemistry and Life Sciences (MicroTAS 2016), Dublin, 2016 (Chemical and Biological Microsystems Society, Washington, DC, 2016), p. 711.

[57] J. Happel and H. Brenner, Low Reynolds Number Hydrodynamics with Special Applications to Particulate Media (Martinus Nijhoff Publishers, The Hague, 1983). 
[58] H. Bruus, Theoretical Microfluidics (Oxford University Press, Oxford, 2008).

[59] J. Lei, P. Glynne-Jones, and M. Hill, Comparing methods for the modelling of boundary-driven streaming in acoustofluidic devices, Microfluid. Nanofluid. 21, 23 (2017).
[60] G. T. Silva and H. Bruus, Acoustic interaction forces between small particles in an ideal fluid, Phys. Rev. E 90, 063007 (2014).

[61] M. W. H. Ley and H. Bruus, Continuum modeling of hydrodynamic particle-particle interactions in microfluidic high-concentration suspensions, Lab Chip 16, 1178 (2016). 